\title{
Discrete Bright-dark Soliton Interaction Dynamic Behaviors for Nonautonomous 2+1-Dimensional Soliton Equation
}

\section{Li Li}

Shenyang Normal University

fajun yu ( $\square$ yufajun888@163.com )

Shenyang Normal University

\section{Research Article}

Keywords: Soliton interaction, Bright dark solution, 2+1-dimensinal Ablowitz-Ladik equation

Posted Date: June 14th, 2021

DOl: https://doi.org/10.21203/rs.3.rs-593464/v1

License: (c) (1) This work is licensed under a Creative Commons Attribution 4.0 International License.

Read Full License 


\title{
Discrete bright-dark soliton interaction dynamic behaviors for nonautonomous 2+1-dimensional soliton equation
}

\author{
Li Li Fajun $\mathrm{Yu} *$ \\ School of Mathematics and Systematic Sciences, Shenyang Normal University, Shenyang 110034, \\ China

\begin{abstract}
The non-autonomous discrete bright-dark soliton solutions(NDBDSSs) of the 2+1-dimensional Ablowitz-Ladik(AL) equation are derived. We analyze the dynamic behaviors and interactions of the obtained 2+1-dimensional NDBDSSs. In this paper, we present two kinds of different methods to control the 2+1-dimensional NDBDSSs. In first method, we can only control the wave propagations through the spatial part, since the time function has not effect in the phase part. In second method, we can control the wave propagations through both the spatial and temporal parts. The different propagation phenomena can also be produced through two kinds of managements. We obtain the novel " $\pi$ "-shape non-autonomous discrete bright soliton solution(NDBSS), the novel "ᄉ"-shape non-autonomous discrete dark soliton solution(NDDSS) and their interaction behaviors. The novel behaviors are considered analytically, which can be applied to the electrical and optical fields.
\end{abstract}

Keywords: Soliton interaction; Bright dark solution; 2+1-dimensinal Ablowitz-Ladik equation

\section{Introduction}

The nonlinear differential-difference equations are appropriate to the particle vibrations in lattices, the ferroelectricity in magnetoelectricity materials and the pulses in biological chains. $[1,2,3]$ The AblowitzLadik (AL) hierarchy has a variety of applications to beam steering in nonlinear waveguide arrays[4], chaos in dispersive numerical schemes[5]. A discrete electrical lattice of modulated waves can be modeled by a generalized discrete nonlinear Schrödinger(NLS) equation, which interpolates between the AL and discrete-self-trapping equations in [6]. The wave transmission properties in one dimensional nonlinear lattices were discussed, which analyze the general dynamical system corresponding to some equations, and include the non-integrable discrete NLS equation and the integrable AL equation in[7].

A lot of nonlinear soliton equations have been solved with some soliton methods, such as the Bäcklund transformation, Darboux transformation(DT), similarity transformation, and so on [8, 9, 10, 11, 12]. For examples, some discrete quasi-periodic solutions of the AL equation are discussed in Refs. $[13,14,15,16]$. Some effective methods of solving the nonlinear soliton equations are considered [17, 18, 19, 20]. And, a discrete rogue-wave solution of Ablowitz-Musslimani equation with parity-time symmetric potential is derived in [21]. The non-autonomous rogue wave, bright, dark and breather solutions of nonlinear GrossPitaevskii(GP) equation are investigated in $[22,23]$. Several non-autonomous dark-bright solutions of the generalized three-coupled GP equations are obtained in [24]. The non-autonomous discrete soliton solutions and their dynamic behaviors in the coupled AL equation are considered in [25, 26]. Some non-autonomous soliton solutions of GP equation are found in optics and BECs [27, 28, 29]. Yan, et al

${ }^{*}$ Corresponding author. Fajun Yu, Tel.: +86 13840329476; fax: +86 24 86505823; E-mail address: yufajun888@163.com 
present some various types of matter-wave solutions and non-autonomous rogue waves in discrete soliton equation $[30,31,32,33,34]$.

The discrete AL equations have very interesting mathematical properties [35, 36], and are investigated in $[37,38,39]$. Yu presents the NDBSSs and their interactions of AL equation in Ref.[40]. Here, we further extend and investigate the 2+1-dimensional NDBDSSs and some dynamic behaviors, which is different from those results in the 1+1-dimensional work. We consider some 2+1-dimensional discrete solutions of the AL equation, and give the wave propagations of 2+1-dimensional NDBDSSs. We also consider several NDBDSSs with the different types of the managements, including the space-part management and the space-time management. In these two cases, sometimes the same phenomena can be achieved, and sometimes the different propagation phenomena can also be produced through the different managements. We study the novel discrete " $\pi$ "-shape NDBSS, "人”-shape NDDSS and their interaction behaviors.

A novel form of 2+1-dimensional discrete similarity transformation is presented in Sec.2. In Sec. 3, we derive two 2+1-dimensional NDBDSSs, then these solutions with space-management are investigated analytically, and we consider their abundant propagation behaviors. We obtain 2+1-dimensional NDBSSs and NDDSSs, including 1-soliton and 2-soliton solutions in Sec. 4 and Sec. 5. Furthermore, the interactions with space-time managements of these solutions are investigated analytically.

\section{A novel discrete similarity transformation for 2+1-dimensional AL equation}

The 2+1-dimensional AL equation can be reduced to the 1+1-dimensional AL equation, which can describe analytically various dynamical regimes of the soliton train in[38]. We here consider the 2+1dimensional variable coefficients AL equation as following form

$$
\begin{aligned}
i \frac{\partial \Psi_{n, m}}{\partial t}+ & \Lambda_{1}(t) \Psi_{n+1, m}+\Lambda_{1}^{*}(t) \Psi_{n-1, m}+\Lambda_{2}(t) \Psi_{n, m+1}+\Lambda_{2}^{*}(t) \Psi_{n, m-1}-4 V(n, m, t) \Psi_{n, m}+i \gamma(t) \Psi_{n, m} \\
& +g(t)\left|\Psi_{n, m}\right|^{2}\left[\Lambda_{1}(t) \Psi_{n+1, m}+\Lambda_{1}^{*}(t) \Psi_{n-1, m}+\Lambda_{2}(t) \Psi_{n, m+1}+\Lambda_{2}^{*}(t) \Psi_{n, m-1}\right]=0
\end{aligned}
$$

where $\Psi_{n, m}(t)$ is the complex field of $n$ and $m$, the $\Lambda_{i}(t)=a_{i}(t)+i b_{i}(t),(i=1,2)$, with $a_{i}(t), b_{i}(t)$ are time functions, $V(n, m, t)$ is the space-time modulated potential, the $g(t)$ represents the time-modulated nonlinearity, and $\gamma(t)$ is the gain or loss term. The 2+1-dimensional AL equation (1) contains many special lattice models, such as the ALH lattice[34], AL lattice [31, 32, 41], discrete Hirota equation[42, 43] and discrete $\mathrm{mKdV}$ equation[44, 45].

We consider a discrete similarity transformation, which can connect the solutions of Eq.(1) with some solutions of the following constant coefficients AL equation

$$
\begin{gathered}
i \frac{\partial u_{n, m}}{\partial \tau}+\nu\left[\lambda_{1}(t) u_{n+1, m}+\lambda_{1}^{*}(t) \Psi_{n-1, m}+\lambda_{2}(t) u_{n, m+1}+\lambda_{2}^{*}(t) u_{n, m-1}-4 u_{n, m}\right] \\
\quad+\frac{\delta}{4}\left|u_{n, m}\right|^{2}\left[\lambda_{1}(t) u_{n+1, m}+\lambda_{1}^{*}(t) u_{n-1, m}+\lambda_{2}(t) u_{n, m+1}+\lambda_{2}^{*}(t) u_{n, m-1}\right]=0
\end{gathered}
$$

where $u_{n, m}(\tau)$ and $\tau=\tau(t), \lambda_{i}=\alpha_{i}+i \beta_{i}$ is complex.

In order to solve Eq.(2), we consider a trivial background. According to the similarity transformations[34, $46,47,48,49]$, we take a different discrete similarity transformation and search for the solution of physical field

$$
\Psi_{n, m}(t)=\rho(t) e^{i \varphi_{n, m}(t)} u_{n, m}(\tau(t)),
$$

with $\rho(t)$ and $\varphi_{n, m}(t)$ are the real functions of the indicated variables.

We substitute the discrete transformation(3) into Eq.(1) and balance the phases of $\Psi_{n, m}(t), \Psi_{n+1, m}(t)$, $\Psi_{n-1, m}(t), \Psi_{n, m+1}(t)$ and $\Psi_{n, m-1}(t)$ in Eq.(1). It is different from the phase of the generalized ALH lattice[34], here the phase is a cubic space polynomial as following 


$$
\varphi_{n, m}(t)=p_{3}(t) n^{3}+q_{3}(t) m^{3}+p_{2}(t) n^{2}+q_{2}(t) m^{2}+p_{1}(t) n+q_{1}(t) m+p_{0}(t) .
$$

Based on the discrete symmetry analysis, we can obtain $p_{3}(t)=0, q_{3}(t)=0, p_{2}(t)=0, q_{2}(t)=0$. So that the phase is a first degree space polynomial in system $(4)$, and the coefficients are some time-functions

$$
\varphi_{n, m}(t)=p_{1}(t) n+q_{1}(t) m+p_{0}(t),
$$

where $p_{0}(t), p_{1}(t)$ and $q_{1}(t)$ are time-functions.

From the discrete similarity transformation (3), we get the following systems

$$
\left\{\begin{array}{l}
\dot{\rho}(t)+\gamma(t) \rho(t)=0 \\
g(t) \rho^{2}(t)=\frac{\delta}{\nu} \\
4 \dot{\tau}(t)-\varphi_{t}(n, m, t)-4 V(n, m, t)=0 \\
\nu \alpha_{1} \dot{\tau}(t)-a_{1}(t) \sin p_{1}(t)+b_{1}(t) \cos p_{1}(t)=0 \\
\nu \beta_{1} \dot{\tau}(t)-b_{1}(t) \sin p_{1}(t)-a_{1}(t) \cos p_{1}(t)=0 \\
\nu \alpha_{2} \dot{\tau}(t)-a_{2}(t) \sin q_{1}(t)+b_{2}(t) \cos q_{1}(t)=0 \\
\nu \beta_{2} \dot{\tau}(t)-b_{2}(t) \sin q_{1}(t)-a_{2}(t) \cos q_{1}(t)=0 \\
\left(\alpha_{1} a_{1}(t)+\beta_{1} b_{1}(t) \sin p_{1}(t)+\left(\beta_{1} a_{1}(t)-\alpha_{1} b_{1}(t)\right) \cos p_{1}(t)=0\right. \\
\left(\alpha_{2} a_{2}(t)+\beta_{2} b_{2}(t)\right) \sin q_{1}(t)+\left(\beta_{2} a_{2}(t)-\alpha_{2} b_{2}(t)\right) \cos q_{1}(t)=0
\end{array}\right.
$$

the functions $\rho(t), \tau(t), q_{1}(t), p_{1}(t)$, the external potential $V(n, m, t)$ and nonlinearity $g(t)$ can be solved in Eq.(6).

According to the fourth and fifth equations in Eq.(6), we have

$$
\begin{aligned}
& \nu \alpha_{1} \dot{\tau}(t)=a_{1}(t) \sin p_{1}(t)-b_{1}(t) \cos p_{1}(t), \\
& \nu \beta_{1} \dot{\tau}(t)=b_{1}(t) \sin p_{1}(t)+a_{1}(t) \cos p_{1}(t),
\end{aligned}
$$

we get the square on both sides of the above two equations, and consider their summations, then obtain the following equation

$$
\nu^{2}\left(\alpha_{1}^{2}+\beta_{1}^{2}\right) \dot{\tau}^{2}(t)=a_{1}^{2}(t)+b_{1}^{2}(t)
$$

The similarity variables $\rho(t), \tau(t), p_{1}(t)$ and $q_{1}(t)$ are presented through solving the complex system (6) in the following forms

$$
\left\{\begin{array}{l}
\rho(t)=\rho_{0} e^{-\int_{k}^{t} \gamma(s) d s}, \\
p_{1}(t)=\tan ^{-1}\left\lceil\frac{\alpha_{1} b_{1}(t)-\beta_{1} a_{1}(t)}{\alpha_{1} a_{1}(t)+\beta_{1} b_{1}(t)}\right], \\
q_{1}(t)=\tan ^{-1}\left\lceil\frac{\alpha_{2} b_{2}(t)-\beta_{2} a_{2}(t)}{\alpha_{2} a_{2}(t)+\beta_{2} b_{2}(t)}\right] \\
\tau(t)=\frac{1}{\nu}\left(\alpha_{i}^{2}+\beta_{i}^{2}\right)^{-\frac{1}{2}} \int_{c}^{t}\left[a_{i}^{2}(s)+b_{i}^{2}(s)\right]^{\frac{1}{2}} d s, i=1,2,
\end{array}\right.
$$

where $c, k$ are two arbitrary constants, $\rho_{0}$ is a constant.

We take a discrete similarity transformation $\Psi_{n, m}(t)=\rho(t) e^{i \varphi_{n, m}(t)} u_{n, m}(\tau(t))$ into Eq.(1), the coefficients of $u_{n, m}(\tau(t))$ and $u_{n, m, \tau}$ are the terms $\rho(t) e^{i \varphi_{n, m}(t)}\left(-\varphi_{n, m, t}-4 V\right)$ and $\rho(t) e^{i \varphi_{n, m}(t)} \dot{\tau}(t)$, then we construct the relations between the terms $u_{n, m}(\tau(t)), u_{n, m, \tau}$ and Eq.(2), and obtain the following system

$$
\frac{\rho \dot{\tau}(t)}{1}=\frac{\rho\left(-\varphi_{n, m, t}-4 V\right)}{-4}=\ldots \ldots
$$

As a consequence, we derive the 3rd equation of Eq.(6)

$$
4 \dot{\tau}(t)-\varphi_{t}(n, m, t)-4 V(n, m, t)=0 .
$$

There are many works of the Gross-Pitaevski equations with (time, space)-modulated potential and nonlinearity, such as the harmonic potential $x^{2}$, the van der Waals law potential $1 / x^{3}$, the harmonic 
potentials. Therefore, we can obtain a linear (time, space)-modulated external potential $V(n, m, t)$ of $n, m$ and the nonlinearity function $g(t)$,

$$
\left\{\begin{array}{l}
g(t)=\frac{\delta}{\nu \rho_{i 0}^{2}} e^{2 \int_{k}^{t} \gamma_{i}(s) d s}, \\
V(n, m, t)=v_{12}(t) n+v_{11} m+v_{10}(t),
\end{array}\right.
$$

where three external potential functions $v_{i j}(n, m, t)$ are given rise to

$$
\left\{\begin{array}{l}
v_{12}(t)=-\frac{\dot{p}_{1}(t)}{4}, \\
v_{11}(t)=-\frac{\dot{q}_{1}(t)}{4} \\
v_{10}(t)=-\frac{\dot{p}_{0}(t)}{4}+\left[\frac{a_{i}^{2}(t)+b_{i}^{2}(t)}{\alpha_{i}^{2}+\delta_{i}^{2}}\right]^{\frac{1}{2}}, \quad i=1,2 .
\end{array}\right.
$$

\section{Sapce-management for 2+1-dimensional non-autonomous dis- crete solutions}

The bright and dark soliton solutions of a AL equation are obtained via the Hirota bilinear method in Ref.[50]. However, the 2+1-dimensional NDBDSSs and their interactions of the AL equation have not been studied, we will analyze the soliton dynamics of Eq.(1) with the management functions of $a_{i}(t), b_{i}(t)$ and $\gamma(t)$. In this section, we can find that the $\tau(t)$ is not effect to the wave propagation, because it only appears in the phase position. So that we can only control the wave propagation through the sapce-management for the 2+1-dimensional NDBDSSs.

Based on the discrete transformation (3) and the dark soliton of Eq.(2), we obtain the 2+1-dimensional one-NDDSS of Eq.(1) with $\delta=1, \epsilon=\nu$

$$
\begin{gathered}
\Psi_{n, m}^{(1)}(t)=2 \rho_{0} e^{\int_{0}^{t}-\gamma(s) d s+i\left[p_{1}(t) n+q_{1}(t) m+p_{0}\right]} \tanh (d n+h m) \sqrt{\frac{\epsilon\left((\sinh (h))^{2}+(\sinh (d))^{2}\right)}{(\operatorname{coth}(d))^{2}(\sinh (h))^{2}+(\operatorname{coth}(h))^{2}(\sinh (d))^{2}} \times} \\
e^{\left(2 i\left(\frac{(\operatorname{csch}(d) \sinh (h)+\operatorname{csch}(h) \sinh (d))^{2}}{(\operatorname{coth}(d))^{2}(\sinh (h))^{2}+(\operatorname{coth}(h))^{2}(\sinh (d))^{2}}-2\right) \epsilon \tau(t)\right)}
\end{gathered}
$$

where $\tau(t)=\frac{1}{\nu}\left(\alpha_{i}^{2}+\beta_{i}^{2}\right)^{-\frac{1}{2}} \int_{c}^{t}\left[a_{i}^{2}(s)+b_{i}^{2}(s)\right]^{\frac{1}{2}} d s, i=1,2$, the variable $\tau(t)$ is solved by Eq.(7) with $\alpha_{i}=1, \beta_{i}=0$, and the $p_{0}(t)$ is an arbitrary time-function.

We consider the complex wave propagation of 2+1-dimensional one-NDDSS(10) with some free parameters $\epsilon=0.1, d=1, h=1, \rho_{0}=1, \alpha_{i}=1, \beta_{i}=0$. When we choose the space-function $m=2 n$ and $\gamma(t)=-0.5 \sin (t) \cos (t)$, the intensity distribution of one-NDDSS(10) is illustrated in Fig.1(a). And, we can find that the amplitude of one-NDDSS is invariant as time increases in Fig.1(b). From Figs 1.(a) and (b), we can see that the amplitude and the width of periodic propagation remain invariable with the increasing distance. In the case of $m=2 n, \gamma(t)=-0.1 s n(2 t, 1)$, a structure of one-NDDSS is known as "Concave paraboloid-like" and is illustrated in Fig.2(c) and (d), which is a novel intensity distribution of one-NDDSS in Eq.(10).

Take using of the discrete transformation (3) and the bright soliton solution of Eq.(2), a 2+1dimensional one-NDBSS of Eq.(1) is derived with $\delta=1, \epsilon=\nu$

$$
\begin{gathered}
\Psi_{n, m}^{(1)}(t)=\rho_{0} e^{\int_{0}^{t}-\gamma(s) d s+i\left[p_{1}(t) n+q_{1}(t) m+p_{0}\right]} \frac{1}{\tanh (d n+h m)} \times \\
2 \sqrt{\frac{\epsilon\left((\sinh (h))^{2}+(\sinh (d))^{2}\right)}{(\operatorname{coth}(d))^{2}(\sinh (h))^{2}+(\operatorname{coth}(h))^{2}(\sinh (d))^{2}}} e^{\left(2 i\left(\frac{(\operatorname{csch}(d) \sinh (h)+c \operatorname{sch}(h) \sinh (d))^{2}}{(\operatorname{coth}(d))^{2}(\sinh (h))^{2}+(\operatorname{coth}(h))^{2}(\sinh (d))^{2}}-2\right) \epsilon \tau(t)\right)} .
\end{gathered}
$$


(b)

(a)

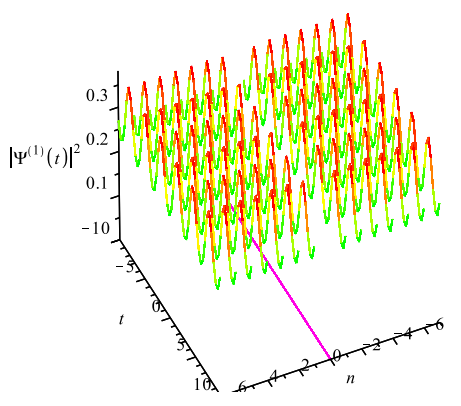

(c)

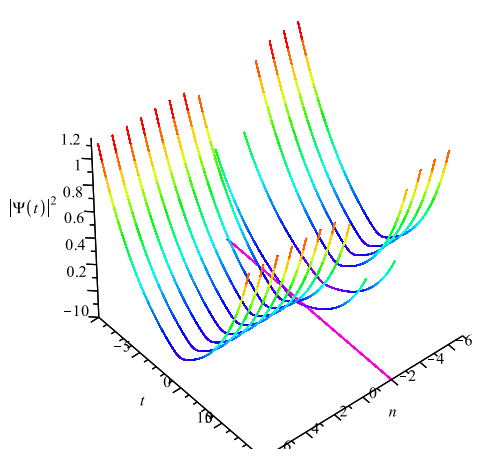

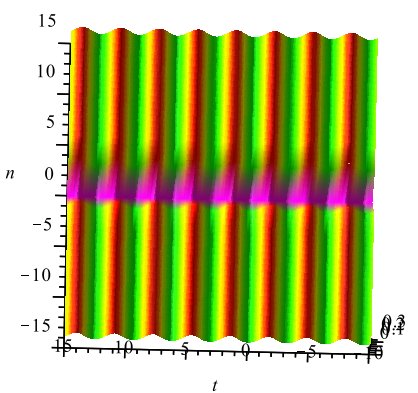

(d)

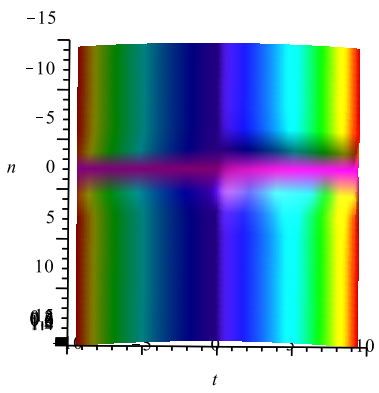

Figure 1: (Color online) (a) the intensity distribution $\left|\Psi_{n, 2 n}^{(1)}(t)\right|$ with $\epsilon=0.1, d=1, h=1, \gamma(t)=$ $-0.5 \sin (t) \cos (t), \rho_{0}=1$ in Eq.(10), (b) the density plot $\left|\Psi_{n, 2 n}^{(1)}(t)\right|$. (c) the intensity distribution $\left|\Psi_{n, 2}^{(1)}(t)\right|$ with $\epsilon=0.1, d=1, h=1, \gamma(t)=-0.1 \operatorname{sn}(2 t, 1), \rho_{0}=1$ in Eq.(10), (d) the density plot of $\left|\Psi_{n, 2 n}^{(1)}(t)\right|$.

We illustrate the wave propagations of 2+1-dimensional one-NDBSS(11) with the specific free parameters $\epsilon=0.1, d=3, h=2,, \rho_{0}=1, \alpha_{i}=1, \beta_{i}=0$. Two specific evolutions of the intensity distributions of one-NDBSS(11) are illustrated in Figs.2(a) and (b) with space-function $m=-1$, and $\gamma(t)=2 \sin (5 t) \cos (5 t)$. Therefore we can find that the amplitude and the width of one-NDBSS remain invariable with the increasing distance in Figs 2.(a) and (b), which can describe the periodic propagation of one-NDBSS. An interesting evolution of one-NDBSS(11) is illustrated in Fig.2(c) and (d) with $m=-1, \gamma(t)=0.1 \operatorname{sn}(2 t, 1)$, such a structure of one-NDBSS is known as "Convex paraboloid-like".

In the Figs.2 (c) and (d), the solution is initially a bright soliton with relatively wide and top. With time, the width of the top does not decrease and the height does not increase. We consider some dynamical behaviors of the discrete bright solution with some parameters, the time evolution of the discrete bright solution may be almost stable in the time propagation with the perturbation. 
(b)

(a)
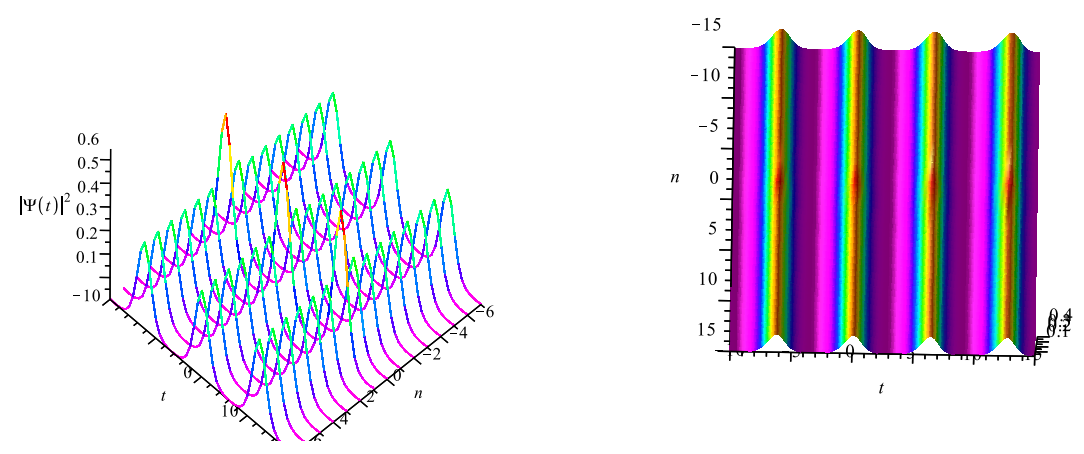

(d)

(c)
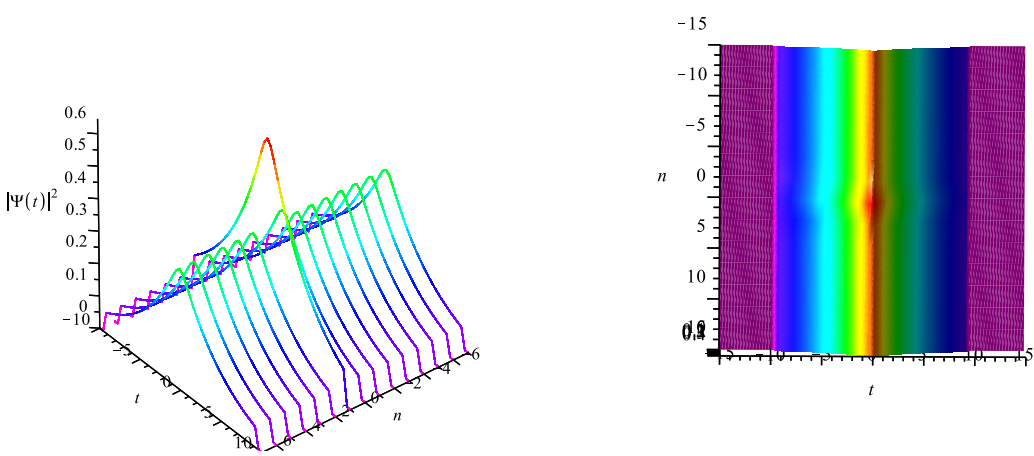

Figure 2: (Color online) (a) the intensity distribution $\left|\Psi_{n,-1}^{(1)}(t)\right|$ with $\epsilon=0.1, d=3, h=2, \gamma(t)=$ $2 \sin (5 t) \cos (5 t), \rho_{0}=1$ in Eq.(11), (b) the density plot of (a). (c) the intensity distribution $\left|\Psi_{n,-1}^{(1)}(t)\right|$ with $\epsilon=0.1, d=3, h=2, \gamma(t)=0.1 \operatorname{sn}(2 t, 1), \rho_{0}=1$ in Eq.(11), (d) the density plot of $(\mathrm{c})$.

\section{2+1-dimensional non-autonomous bright solutions and inter- actions with space-time management}

We consider the controllable interactions of 2+1-dimensional NDBSSs, which are different from the results in our previous work. And we will analyze the 2+1-dimensional NDBSS and their dynamics of Eq.(1) with the effects of $a_{i}(t), b_{i}(t)$ and $\gamma(t)$. The obtained results might give some theoretical analysis in controllable soliton.

\subsection{2+1-dimensional one-NDBSS with space-time management}

The N-bright soliton solutions are obtained in a discrete AL equation[51]. We can derive 2+1dimensional one-NDBSS $\Psi_{n, m}^{(1)}(t)$ from the above system in section 2 , and the $\Psi_{n, m}^{(1)}(t)$ is a function of the discrete spaces $n$ and $m$ as following

$$
\Psi_{n, m}^{(1)}(t)=\rho_{0} e^{-\int_{0}^{t} \gamma(s) d s+i\left[p_{1}(t) n+q_{1}(t) m+p_{0}+\right]} i^{n+m} \frac{1}{2 \sqrt{|a|}} e^{(-4 \epsilon i)} \operatorname{sech}\left(\frac{\left(\theta+\theta^{*}+\ln (|a|)\right.}{2}\right),
$$


where $a=1 / 4 \frac{\mathrm{e}^{\iota_{1}+\iota_{1}^{*}}}{\left(-1+\mathrm{e}^{\iota_{1}+\iota_{1}^{*}}\right)^{2} \epsilon}, \theta=k \tau(t)+\iota_{1} n+\iota_{2} m+\theta_{0}, k=-2 \mathrm{e}^{-\iota_{1}}\left(i+\mathrm{e}^{\iota_{1}}\right)^{2} \epsilon, \tau(t)=\frac{1}{\nu}\left(\alpha_{i}^{2}+\right.$ $\left.\beta_{i}^{2}\right)^{-\frac{1}{2}} \int_{c}^{t}\left[a_{i}^{2}(s)+b_{i}^{2}(s)\right]^{\frac{1}{2}} d s, i=1,2$, the $\tau(t)$ is given in Eq.(7) with $\alpha_{i}=1, \beta_{i}=0$, the $\iota_{1}$ and $\iota_{2}$ are two constants, and $p_{0}(t)$ is a time-function.

We illustrate the wave propagation of 2+1-dimensional one-NDBSS(12) with $\epsilon=1, \iota_{1}=0.5+0.1 i, \iota_{1}=$ $\iota_{2}, \gamma(t)=0.1 \cos (t), \rho_{0}=1$. Fig. 3(a) shows the 2+1-dimensional one-NDBSS in Eq.(12) with timemanagement function $\tau(t)=0.05 t^{2}$. Fig.3(b) shows the 2+1-dimensional one-NDBSS in Eq.(12) with space-management function $m=n^{2}$. Figs 3.(a) and (b) describe the propagations of the 2+1-dimensional one-NDBSSs, such a structure of the NDBSS is known as "Parabola-like".

(a)

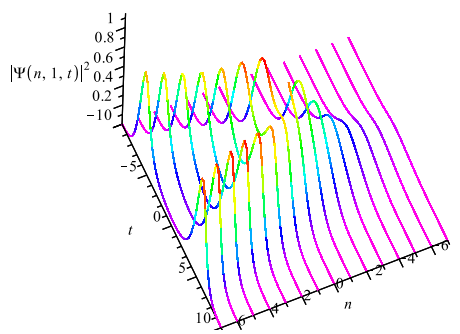

(c)

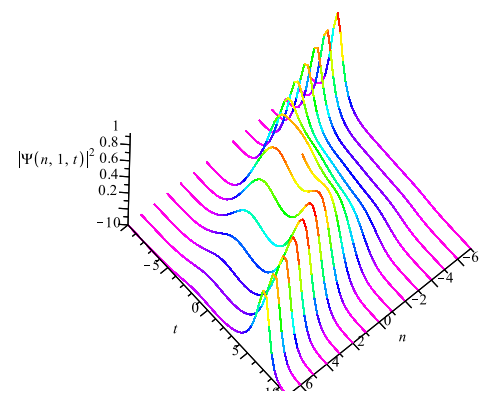

(b)

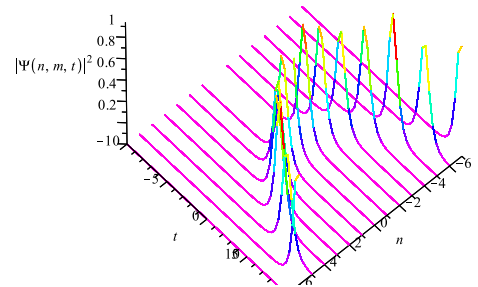

(d)

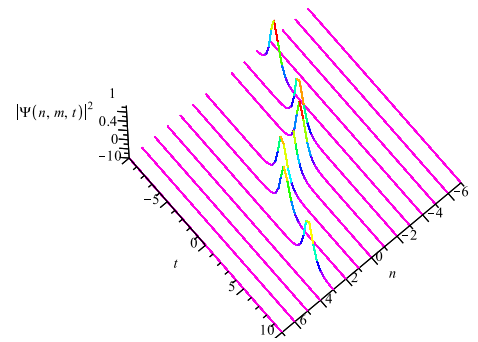

Figure 3: (Color online) Profiles of the intensity distribution (a) $\left|\Psi_{n, 1}^{(1)}(t)\right|$ with $\tau(t)=0.05 t^{2}, \epsilon=$ $1, \iota_{1}=0.5+0.1 i, \iota_{1}=\iota_{2}, \gamma(t)=0.1 \cos (t), \rho_{0}=1$ in Eq.(12); (b) $\left|\Psi_{n, m}^{(1)}(t)\right|$ with $\tau(t)=t, m=n^{2}$, $\epsilon=1, \iota_{1}=0.5+0.1 i, \iota_{1}=\iota_{2}, \gamma(t)=0.1 \cos (t), \rho_{0}=1$ in Eq.(12); (c) $\left|\Psi_{n, 1}^{(1)}(t)\right|$ with $\tau(t)=0.05 t^{3}$, $\epsilon=1, \iota_{1}=0.5+0.1 i, \iota_{1}=\iota_{2}, \gamma(t)=0.1 \cos (t), \rho_{0}=1$ in Eq.(12); (d) $\left|\Psi_{n, m}^{(1)}(t)\right|$ with $\tau(t)=t, m=n^{3}$, $\epsilon=1, \iota_{1}=0.5+0.1 i, \iota_{1}=\iota_{2}, \gamma(t)=0.1 \cos (t), \rho_{0}=1$ in Eq. $(12)$.

Fig.3 (c) depicts the dynamical behavior of the 2+1-dimensional one-NDBSS(12) with the timemanagement $\tau(t)=0.05 t^{3}$. Fig.3 (d) illustrates the intensity distribution of the 2+1-dimensional one$\operatorname{NDBSS}(12)$ with space-management. So, we adjust the coefficients $\tau(t)=t, m=n^{3}$ given by Eq.(12), then the evolution of the intensity distribution of the 2+1-dimensional one-NDBSS is changed (see Fig.3 (c) (d)), and the amplitude of the one-NDBSS remains the same, but its central position oscillates periodically with the increasing distance in Fig. 3(d). Such a structure of the 2+1-dimensional oneNDBSS is known as "Snake-like".

The parameters $a_{i}(t), b_{i}(t)$ and $\gamma(t)$ are the important elements of effect on the amplitudes and shapes of the 2+1-dimensional one-NDBSS in Fig 3. Figs. 3(a) and (c) present two kinds of especial solution structures with the "Parabola-like" and "Snake-like". Figs. 3(b) and (d) show the discrete peaks of oneNDBSS with certain amplitude, width and velocity through space-management and time-management, respectively. 


\subsection{2+1-dimensional two-NDBSS with space-time managements}

In this section, we consider how to control the $2+1$-dimensional discrete soliton interactions with the functions $a_{i}(t), b_{i}(t)$ and $\gamma(t)$. If we choose $\alpha_{i}=1, \beta_{i}=0$ and $\lambda_{i}=1$, the $\varphi_{n, m}(t)$ is a linear function of the discrete spaces $n$ and $m$. We derive the $2+1$-dimensional two-NDBSS $\Psi_{n, m}^{(2)}(t)$ of Eq. (1) as following

$$
\Psi_{n, m}^{(2)}(t)=\rho_{0} e^{-\int_{0}^{t} \gamma(s) d s+i\left[p_{1}(t) n+q_{1}(t) m+p_{0}\right]} .
$$

$$
\left[(i)^{n+m} \frac{e^{\theta_{1}}+e^{\theta_{2}}+a_{31} e^{\theta_{1}+\theta_{2}+\theta_{1}^{*}}+a_{32} e^{\theta_{1}+\theta_{2}+\theta_{2}^{*}}}{1+a_{11} e^{\theta_{1}+\theta_{1}^{*}}+a_{12} e^{\theta_{1}+\theta_{2}^{*}}+a_{21} e^{\theta_{2}+\theta_{1}^{*}}+a_{22} e^{\theta_{2}+\theta_{2}^{*}}+a_{4} e^{\theta_{1}+\theta_{1}^{*}+\theta_{2}+\theta_{2}^{*}}}\right],
$$

with $\theta_{i}=k_{i} \tau(t)+\alpha_{j 1} n+\alpha_{j 2} m+\theta_{0}, \alpha_{j 1}=\alpha_{j 2}, k_{j}=-2 \mathrm{e}^{-\alpha_{j 1}}\left(i+\mathrm{e}^{\alpha_{j 1}}\right)^{2} \epsilon, a_{1 j}=1 / 4 \frac{\mathrm{e}^{\alpha_{1 j}+\alpha_{j 1}^{*}}}{\left(-1+\mathrm{e}^{\alpha_{1 j}+\alpha_{j 1}^{*}}\right)^{2} \epsilon}$, $a_{2 j}=1 / 4 \frac{\mathrm{e}^{\alpha_{2 j}+\alpha_{j 2}^{*}}}{\left(-1+\mathrm{e}^{\alpha_{2 j}+\alpha_{j 2}^{*}}\right)^{2} \epsilon}, a_{3 j}=1 / 4 \frac{\mathrm{e}^{2 \alpha_{1 j}^{*}\left(\mathrm{e}^{\alpha_{1 j}}-\mathrm{e}^{\alpha_{2 j}}\right)^{2}}}{\left(-1+\mathrm{e}^{\alpha_{11}+\alpha_{j 1}^{*}}\right)^{2}\left(-1+\mathrm{e}^{\alpha_{21}+\alpha_{j 1}^{*}}\right)^{2} \epsilon}$,

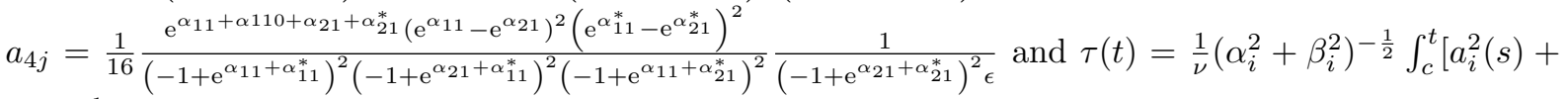
$\left.b_{i}^{2}(s)\right]^{\frac{1}{2}} d s, i=1,2$.

We illustrate the wave structures of the obtained 2+1-dimensional two-NDBSS(13) with $a_{i}(t), b_{i}(t), \gamma(t)$ and $\rho_{0}=1, \alpha_{i}=1, \beta_{i}=0$. It follows from Figs. 4(a), (b) and (c) that the interactions between two NDBSSs are elastic collisions with different velocities. Fig. 4 (d) shows the linear type propagation of two-NDBSS, which is invariant during the propagation and is parallel line and has not the interaction.

Especially, it is interesting to note that a novel $\pi$-shape two-NDBSS is presented in Fig. 4 (c). And, two NDBSSs encounter with each other and have the interactions "Bi-opposite-parabola-like" in Figs.4 (a) and (b). In Fig. 4(a), two NDBSSs encounter each other, which are controlled with time-controllable function $\tau(t)=0.1 t^{2}$. In Fig. 4(b), the interaction of two NDBSSs is controlled with space-controllable function $m=n^{2}$. Figs.4 (a) and (b) show the interactions between two NDBSSs with two different controllable methods, but their shapes are the same except for some phase shifts.

The processes of the interaction between two solitons with different controllable methods and different controllable functions are shown in Fig.4. A novel $\pi$-shape two-NDBSS appears in Fig. 4(c), it appears an interesting phenomenon in the AL equation. While, the two-NDBSSs can transmit in paralleled waves with the same shape and velocity in Fig.4(d). The parallel between nonlinear guided wave phenomena can demonstrate in optics.

\section{2+1-dimensional non-autonomous dark solutions and interac- tions with space-time management}

In this section, we consider the soliton dynamics of Eq.(1) with time-, space- and space-time-managements based on some 2+1-dimensional NDDSSs.

\section{$5.12+1$-dimensional one-NDDSS and management}

Some dark soliton solutions of Eq.(2) are obtained in [51]. We take the discrete transformation (3) and the dark soliton solution of Eq.(2), and present the $2+1$-dimensional one-NDDSS $\Psi_{n, m}^{(1)}(t)$ of Eq. (1) with $\alpha_{i}=1, \beta_{i}=0, \lambda_{i}=1$ as following

$$
\Psi_{n, m}^{(1)}(t)=\rho_{0} e^{-\int_{0}^{t} \gamma(s) d s+i\left[p_{1}(t) n+q_{1}(t) m+p_{0}\right]}(i)^{n+m} \mu e^{-4 i \epsilon \tau(t)} \tanh \left(\frac{k \tau(t)+\iota_{1} n+\iota_{2} m+\theta_{0}}{2}\right),
$$

where $\delta=-1, \theta=k \tau(t)+\iota_{1} n+\iota_{2} m+\theta_{0}, \iota_{1}=\iota_{2}, k=-8 \frac{\left(-1+\mathrm{e}^{\iota} 1\right) \epsilon}{1+\mathrm{e}^{\iota} 1}, \mu=2 \frac{\left(-1+\mathrm{e}^{\iota 1}\right) \sqrt{|\epsilon|}}{1+\mathrm{e}^{\iota} 1}, \tau(t)=$ $\frac{1}{\nu} \int_{c}^{t}\left[a_{i}^{2}(s)+b_{i}^{2}(s)\right]^{\frac{1}{2}} d s, i=1,2$, the $\varphi_{n, m}(t)=p_{1}(t) n+q_{1}(t) m+p_{0}(t), p_{1}(t)$ in Eq. $(9)$, and $p_{0}(t)$ is a time-function. 
(a)

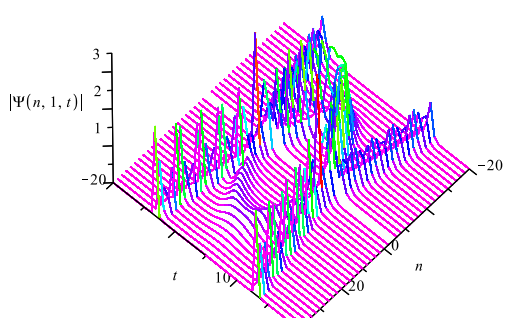

(c)

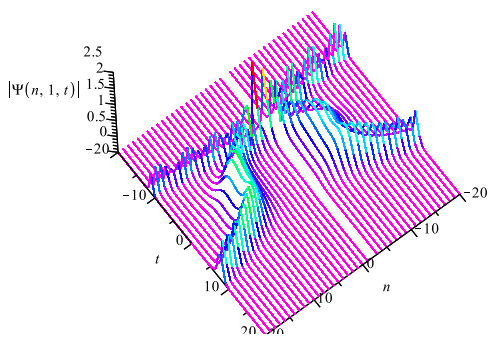

(b)

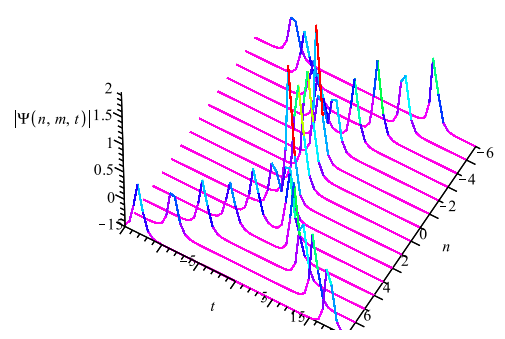

(d)

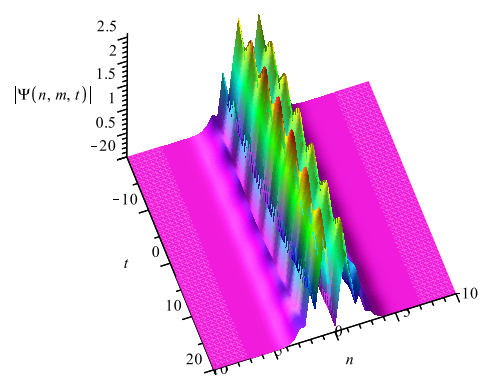

Figure 4: (Color online) The intensity distributions (a) $\left|\Psi_{n, 1}^{(1)}(t)\right|$ with $\tau(t)=0.1 t^{2}, \epsilon=1, \alpha_{11}=0.5-$ $3 i, \alpha_{11}=\alpha_{12}, \alpha_{21}=\alpha_{22}=1, \gamma(t)=0.1 \cos (t), \rho_{0}=1$ in Eq.(13), (b) $\left|\Psi_{n, m}^{(1)}(t)\right|$ with $\tau(t)=0.1 t^{2}, m=n^{2}$, $\epsilon=1, \alpha_{11}=0.5-3 i, \alpha_{11}=\alpha_{12}, \alpha_{21}=\alpha_{22}=0.6, \gamma(t)=0.1 \cos (t), \rho_{0}=1$ in Eq.(13), (c) $\left|\Psi_{n, 1}^{(1)}(t)\right|$ with $\tau(t)=0.05 t^{3}+2, \epsilon=1, \alpha_{11}=0.5-3 i, \alpha_{11}=\alpha_{12}, \alpha_{21}=\alpha_{22}=0.6, \gamma(t)=0.1 \cos (t), \rho_{0}=1$ in Eq.(13), (d) $\left|\Psi_{n, m}^{(1)}(t)\right|$ with $\tau(t)=0.01 t, m=n^{3}+1, \epsilon=1, \alpha_{11}=0.5-3 i, \alpha_{11}=\alpha_{12}, \alpha_{21}=\alpha_{22}=0.6, \gamma(t)=$ $0.1 \cos (t), \rho_{0}=1$ in Eq.(13).

We take the functions $a_{i}(t), b_{i}(t)$ and $\gamma(t)$ to control the soliton evolutions with time- and spacemanagements. The intensity distributions of 2+1-dimensional one-NDDSS(14) are illustrated with $\epsilon=$ $1, \iota_{1}=0.5+0.1 i, \iota_{1}=\iota_{2}, \rho_{0}=1$ in Fig. 5. Then, we adjust the functions $a_{i}(t), b_{i}(t)$ and $\gamma(t)$ in Eq.(14), and the 2+1-dimensional one-NDDSS is changed with time-management in Figs. 5 (a) and (c). Moreover, the 2+1-dimensional one-NDDSS can be observed with space-management in Figs. 5 (b) and (d). A novel structure known as "Parabola-like" of one-NDDSS appears in Figs.5 (a) and (b), a structure known as "Snake-like" appears in Figs.5 (c) and (d), their amplitudes and widths remain invariable, but the central positions oscillate periodically. We can see that the amplitudes and shapes of one-NDDSSs can be influenced by $a_{i}(t)$ and $b_{i}(t)$ in Fig. 5 .

\subsection{2+1-dimensional two-NDDSS and space-time management}

In this section, we consider two kinds of management schemes, one is directly to control the wave propagation with time-management. The other is firstly to control the wave propagation with spacemanagement, then to control the wave propagation with time-management of the functions $a_{i}(t), b_{i}(t)$ and $\gamma(t)$. Based on the two-dark soliton solution with $\alpha=1, \beta=0$ and $\lambda=1$, the $2+1$-dimensional two-NDDSS $\Psi_{n, m}^{(2)}(t)$ is given as following 
(a)

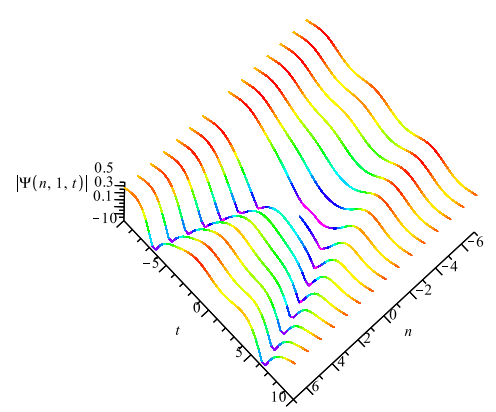

(c)

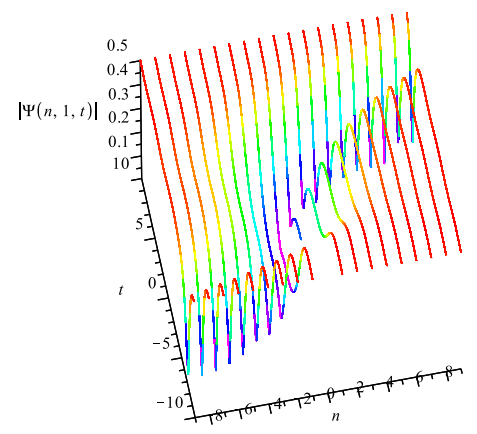

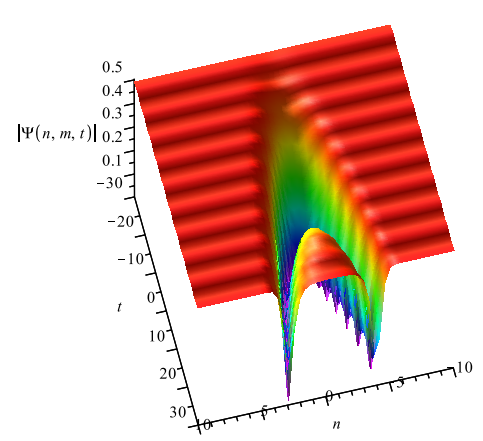

(d)

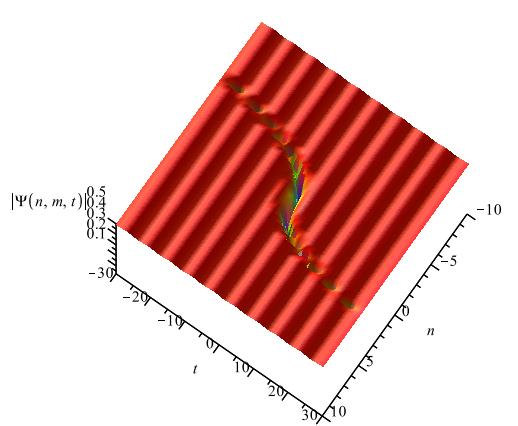

Figure 5: (Color online) Profiles of the intensity distribution (a) $\left|\Psi_{n, 1}^{(1)}(t)\right|$ with $\tau(t)=0.05 t^{2}, \epsilon=$ $1, \iota_{1}=0.5+0.1 i, \iota_{1}=\iota_{2}, \gamma(t)=0.1 \cos (t), \rho_{0}=1$ in Eq.(14), (b) $\left|\Psi_{n, m}^{(1)}(t)\right|$ with $\tau(t)=0.1 t, m=n^{2}$, $\epsilon=1, \iota_{1}=0.5+0.1 i, \iota_{1}=\iota_{2}, \gamma(t)=0.1 \cos (t), \rho_{0}=1$ in Eq.(14), (c) $\left|\Psi_{n, 1}^{(1)}(t)\right|$ with $\tau(t)=0.05 t^{3}$, $\epsilon=1, \iota_{1}=0.5+0.1 i, \iota_{1}=\iota_{2}, \gamma(t)=0.01 \cos (t), \rho_{0}=1$ in Eq.(14), (d) $\left|\Psi_{n, m}^{(1)}(t)\right|$ with $\tau(t)=t, m=n^{3}$, $\epsilon=1, \iota_{1}=0.5+0.1 i, \iota_{1}=\iota_{2}, \gamma(t)=0.01 \cos (t), \rho_{0}=1$ in Eq.(14).

$$
\Psi_{n, m}^{(2)}(t)=\rho_{0} e^{-\int_{0}^{t} \gamma(s) d s+i\left[p_{1}(t) n+q_{1}(t) m+p_{0}\right]}\left[(i)^{n+m} \frac{\mu e^{-4 \epsilon i \tau(t)}\left(1-e^{\theta_{1}+i \eta_{1}}-e^{\theta_{2}+i \eta_{2}}+a e^{\theta_{1}+\theta_{2}+i \eta_{1}+i \eta_{2}}\right)}{1+e^{\theta_{1}}+e^{\theta_{2}}+a e^{\theta_{1}+\theta_{2}}}\right],
$$

where $\theta_{j}=k_{j} \tau(t)+\iota_{j 1} n+\iota_{j 2} m+\theta_{0}, \iota_{j 1}=\iota_{j 2}, \iota_{j 1}=\ln \left(\frac{4 \epsilon+\mu^{2} \cos \left(\eta_{j}\right)+\mu \sqrt{\left(\cos \left(\eta_{j}\right)+1\right)\left(-\mu^{2}+\mu^{2} \cos \left(\eta_{j}\right)+8 \epsilon\right)}}{-\mu^{2}+4 \epsilon}\right)$, $k_{j}=1 / 2 \mathrm{e}^{-\iota_{j 1}}\left(-1+\mathrm{e}^{2 \iota_{j 1}}\right)\left(\mu^{2}-4 \epsilon\right), a=-\frac{\left(\mathrm{e}^{\iota_{11}}-\mathrm{e}^{\iota_{21}}\right) \sin \left(\eta_{1} / 2-\eta_{2} / 2\right)}{\left(-1+\mathrm{e}^{\iota_{11}+\iota_{1} 2}\right)^{2} \sin \left(\eta_{1} / 2+\eta_{2} / 2\right)}$ and $\tau(t)=\int_{c_{2}}^{t}\left[a_{i}^{2}(s)+b_{i}^{2}(s)\right]^{\frac{1}{2}} d s$.

The shape of 2+1-dimensional two-NDDSS can be influenced by the functions $a_{i}(t), b_{i}(t), \gamma(t)$ in Fig. (6), Figs. 6 (a) and (b) show the interactions with the different choices of $\tau(t)=0.1 t^{2}+1$ and $\gamma(t)=0.1 \cos (t)$. Fig.6 (a) presents a novel Bi-parabolic-shape two-NDDSS, which is interesting to note that the amplitude of the collision point also oscillates periodically. A novel Bi-snake-like shape solution appears in Fig.6 (c) and (d), which shows the peaks of two parabolic-lines with the different methods of $\tau(t)=0.05 t^{3}+1$ and $\gamma(t)=0.1 \cos (t)$. We find that the 2+1-dimensional two-NDDSSs can be controlled through the different time-managements, and their velocities are invariant in Fig.6.

Next, we will firstly control the wave propagation with space-management, then control the wave propagation with time-management. Fig. 7 shows the interactions between 2+1-dimensional two-NDDSSs with functions $a_{i}(t), b_{i}(t)$ and $\gamma(t)$. Fig. 7 (a) describes the interaction propagations between two parabolic-type NDDSSs with space-management $m=n^{2}$, then it can be controlled the wave propa- 
(b)

(a)

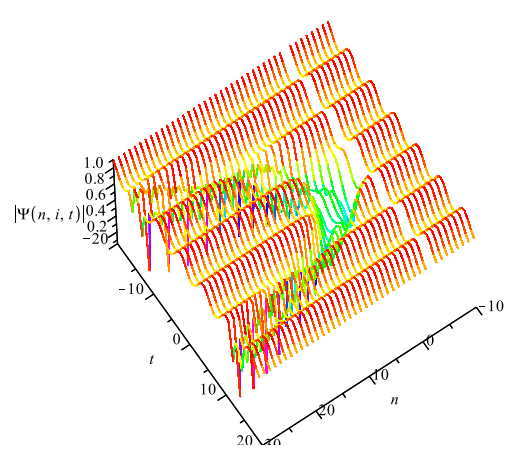

(c)

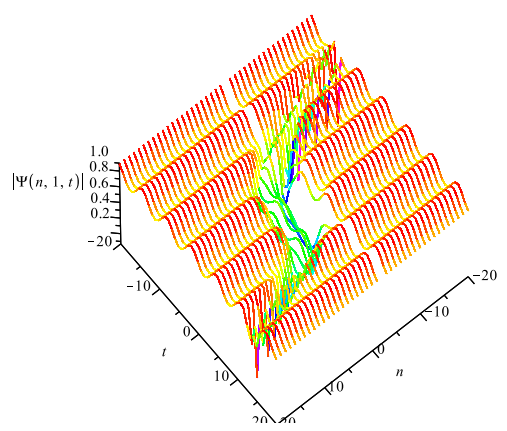

$-20$

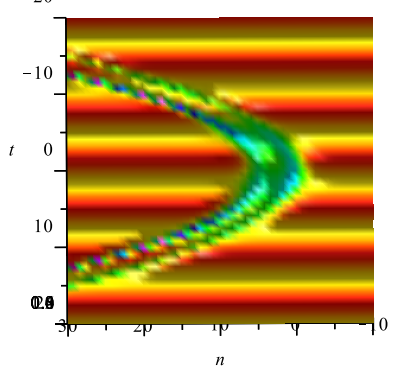

(d)

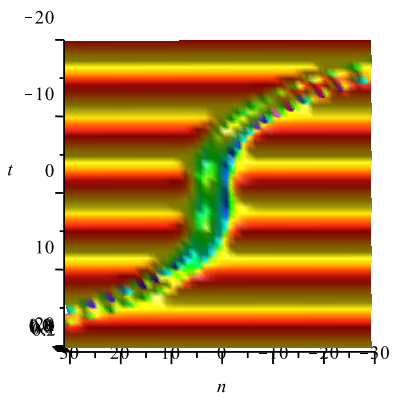

Figure 6: (Color online) Profiles of (a) the intensity distribution $\left|\Psi_{n, 1}^{(2)}(t)\right|$ with $\tau(t)=0.1 t^{2}+1, \epsilon=$ $0.5, \theta_{1}=0, \theta_{2}=\pi / 2, \nu=1, \iota_{j 1}=\iota_{j 2}, \gamma(t)=0.1 \cos (t), \rho_{0}=1$ in Eq.(15), (b) the density plot of $\left|\Psi_{n, 1}^{(2)}(t)\right|$. (c) the intensity distribution $\left|\Psi_{n, 1}^{(2)}(t)\right|$ with $\tau(t)=0.05 t^{3}+1, \epsilon=0.1, \theta_{1}=0, \theta_{2}=\pi / 2, \nu=1, \iota_{j 1}=$ $\iota_{j 2}, \gamma(t)=0.1 \cos (t), \rho_{0}=1$ in Eq.(15), (d) the density plot of $\left|\Psi_{n, 1}^{(2)}(t)\right|$.

gation with time-management $\tau(t)=t^{2}+2$. It is interesting to note that the 2+1-dimensional twoNDDSS has the shape of Bi-opposite-V-like, but it has not the phenomena of interaction in Fig.7 (a). Fig. 7 (c) presents a novel "人"-shape NDDSS with space-management $m=2 n^{2}$ and time-management $\tau(t)=0.05 t^{3}$.

Fig.7 shows that the interactions between two NDDSSs with different controllable functions and methods. The phenomena of elastic collision are happened in Figs. $7(\mathrm{c})$ and (d), but there are not the phenomena of interaction in Figs. 7(a) and (b). Figs.7 (a) and (c) show the interactions with the different space-management and time-management, respectively. In this work, we hope that the obtained non-autonomous soliton dynamics have some theoretical guidance meanings in electrical and optical fields.

\section{Conclusions}

In this paper, the 2+1-dimensional NDBDSSs and interaction behaviors of the AL equation are analyzed, and some novel spatial-temporal structures of 2+1-dimensional NDSSs are found. We consider 
(b)
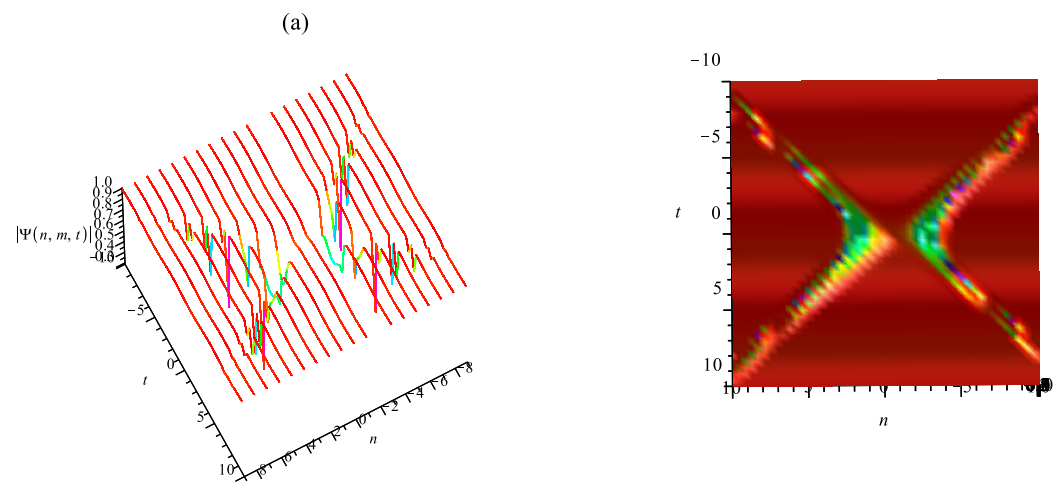

(d)

(c)
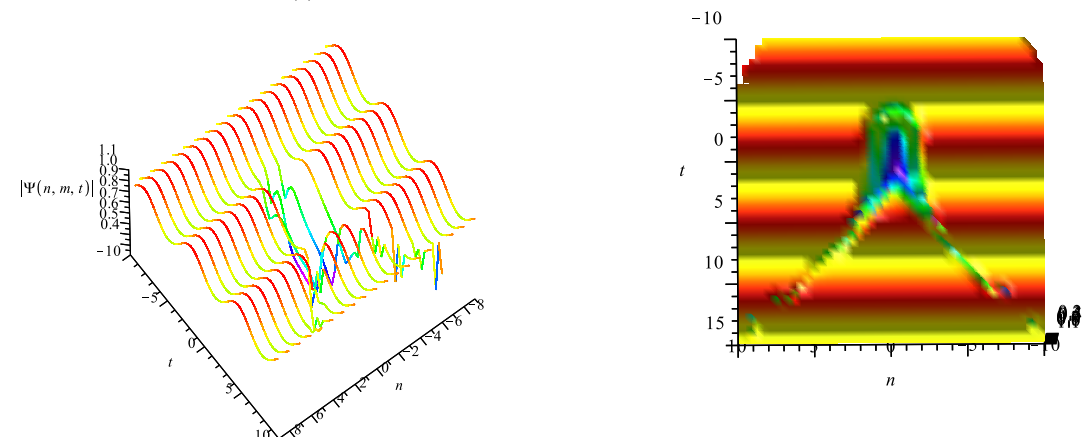

Figure 7: (Color online) Profiles of (a) the intensity distribution $\left|\Psi_{n, m}^{(2)}(t)\right|$ with $m=n^{2}, \tau(t)=t^{2}+2$, $\epsilon=0.1, \theta_{1}=0, \theta_{2}=\pi / 2, \nu=1, \iota_{j 1}=\iota_{j 2}, \gamma(t)=0.1 \cos (t), \rho_{0}=1$ in Eq.(15), (b) the density plot of $\left|\Psi_{n, m}^{(2)}(t)\right|$. (c) the intensity distribution $\left|\Psi_{n, m}^{(2)}(t)\right|$ with $m=2 n^{2}, \tau(t)=0.05 t^{3}, \epsilon=0.5, \theta_{1}=0, \theta_{2}=$ $\pi / 2, \nu=1, \iota_{j 1}=\iota_{j 2}, \gamma(t)=0.1 \cos (t), \rho_{0}=1$ in Eq.(15), (d) the density plot of $\left|\Psi_{n, m}^{(2)}(t)\right|$.

several 2+1-dimensional NDBDSSs with the different managements, including the space-management and the space-time management. We study the novel " $\pi$ "-shape NDBSS, novel "人"-shape NDDSS and their interaction behaviors. The novel phenomena and behaviors are considered analytically in NDSSs, which can be applied to some electrical and optical fields.

\section{Data availability statement}

The data that support the findings of this article are available from the corresponding author, upon reasonable request.

\section{Declaration of competing interest}

The authors declare that they have no known competing financial interests or personal relationships that could have appeared to influence the work reported in this paper. 


\section{Acknowledgments}

This work was sponsored by the Natural Science Foundation of Liaoning Province china(Grant No. 2019ZD0482), Liaoning Baiqianwan Talents Program(2019921075) and Shenyang Normal university major incubation project, China (Grant No. ZD201806).

\section{References}

[1] Enrico, Fermi., Pasta, J., Ulam, S.: Collected papers of enrico ferm, Chicago, IL,Chicago Press, p. 978 (1965).

[2] Levi, D., Yamilov, R.I.: Conditions for the existence of higher symmetries of evolutionary equations on the lattice. J. Math. Phys 38, 6648-6674 (1997).

[3] Sokolov, V.V., Shabat, A.B.: Classification of integrable evolution equations. Sov. Sci. Rev. C: Math Phys Rev 4, 221-280 (1984).

[4] Aceves, A.B., Angelis, C.D., Peschel, T., Muschall, R., Lederer, F., Trillo, S. and Wabnitz, S.: Discrete self-trapping, soliton interactions, and beam steering in nonlinear waveguide arrays. Phys. Rev. E 53, 1172 (1996).

[5] Calini, A., Ercolani, N.M., McLaughlin, D.W. and Schober, C.M.: Analysis of Numerically Induced Chaos in the Nonlinear Schrödinger Equation. Phys. D 89, 227-260 (1996).

[6] Marquie, P., Bilbault, J.M., Remoissenet, M.: Observation of nonlinear localized modes in an electrical lattice. Phys. Rev. E 51, 6127 (1995).

[7] Hennig, D., Tsironis, G.P.: Wave transmission in nonlinear lattices. Phys. Rep 307, 333-342 (1999).

[8] Ablowitz, M.J., Ladik, J.: Nonlinear differential-difference equations and fourier-analysis. J. Math. Phys 17, 1011-1018 (1976).

[9] Ablowitz, M.J., Ladik, J.: A nonlinear difference scheme and inverse scattering. Stud. Appl. Math 55 213-229 (1976).

[10] Kako, F., Mugibayashi, N.: Complete Integrability of General Nonlinear Differential-Difference Equations Solvable by the Inverse Method. Prog. Theor.Phys 61, 776-790 (1979).

[11] Chowdhury, A.R., Mahato, G.: A Darboux-Bäcklund transformation associated with a discrete nonlinear Schrödinger equation. Lett. Math. Phys 7, 313-317 (1983).

[12] Amaral, L., Behar, M., Maciel, A., Saitovitch, H.: Anomalous temperature behaviour of the electric field gradients in an In semiconductor compound. Phys. Lett. A 102, 45-48 (1984).

[13] Bogolyubov, N.N., Prikarpatskii, A.K., Samoilenko, V.G.: Discrete periodic problem for the modified nonlinear Korteweg-de Vries equation. Sov. Phys. Dokl 26, 490-492 (1981).

[14] Bogolyubov, N.N., Prikarpatskii, A.K.: The inverse periodic problem for a discrete approximation of a nonlinear Schrrödinger equation. Sov. Phys. Dokl 27, 113 (1982).

[15] Ahmad, S., Chowdhury, A.R.: The quasiperiodic solutions to the discrete nonlinear Schrödinger equation. J. Math. Phys 28, 134-137 (1987).

[16] Ahmad, S., Chowdhury, A.R.: On the Quasiperiodic Solutions to the Discrete Nonlinear Schrödinger Equation. J. Phys. A 20, 293-303 (1987).

[17] Wang, D.S., Wei, X.Q.: Integrability and exact solutions of a two-component Korteweg-de Vries system. Appl. Math. Lett 51, 60-67 (2016). 
[18] Wang, D.S., Yin, Y.B.: Symmetry analysis and reductions of the two-dimensional generalized Benney system via geometric approach. Compu. Math. Appl 71, 748-757 (2016).

[19] Christodoulides, D.N., Joseph, R.J.: Discrete self-focusing in nonlinear arrays of coupled waveguides. Opt. Lett 193, 794-796 (1988).

[20] Kevrekidis, P.G., Rasmussen, K.O., Bishop, A.R.: The discrete nonlinear Schrodinger equation: a survey of recent results. Int. J. Mod. Phys. B 15, 2833 (2001).

[21] Yu, F.J.: Dynamics of nonautonomous discrete rogue wave solutions for an Ablowitz-Musslimani equation with PT-symmetric potential. Chaos 27, 023108 (2017).

[22] Yu, F.J.: Localized analytical solutions and numerically stabilities of generalized Gross-Pitaevskii $(\mathrm{GP}(\mathrm{p}, \mathrm{q}))$ equation with specific external potentials. App. Math. Lett 85, 1-7 (2018) .

[23] Li, L., Yu, F.J.: Non-autonomous multi-rogue waves for spin-1 coupled nonlinear Gross-Pitaevskii equation and management by external potentials. SCI REP-UK 7, 10638 ( 2017).

[24] Yu, F.J., Li, L.: Vector dark and bright soliton wave solutions and collisions for spin-1 Bose-Einstein condensate. Nonlinear Dynam 87, 2697-2713 (2017).

[25] Li, L., Yu, F.J.: Discrete bright-dark soliton solutions and parameters controlling for the coupled Ablowitz-Ladik equation. Nonlinear Dynam 89, 2403-2414 ( 2017).

[26] Yu F,J., Feng, S.: Explicit solution and Darboux transformation for a new discrete integrable soliton hierarchy with 4 Lax pairs. Math. Method. Appl. Sci 40, 5515-5525 (2017).

[27] Serkin, V.N., Hasegawa, A., Belyaeva, T.L.: Nonautonomous solitons in external potentials. Phys. Rev. Lett 98, 074102 (2007).

[28] Serkin, V.N., Hasegawa, A.: Novel soliton solutions of the nonlinear Schrödinger equation model. Phys. Rev. Lett 85, 4502 (2000).

[29] Serkin, V.N., Hasegawa, A., Belyaeva, T.L.: Solitary waves in nonautonomous nonlinear and dispersive systems: nonautonomous solitons, J. Mode. Optic 57, 1456-1472 (2010).

[30] Yan, Z.Y., Zhang, X.F., Liu, W.M.: Nonautonomous matter waves in a waveguide. Phys. Rev. A 84 023627 (2011).

[31] Eilbeck, J.C., Lomdahl, P.S., Scott, A.C.: Soliton structure in crystalline acetanilide. Phys. D 16, 318-338 (1985).

[32] Ablowitz, M.J., Ladik, J.F.: A nonlinear difference scheme and inverse scattering. Stud. Appl. Math 55, 213-229 (1976).

[33] Ankiewicz, A., Akhmediev, N., Soto-Crespo, J.M.: Discrete rogue waves of the Ablowitz-Ladik and Hirota equations. Phys. Rev. E 82, 026602 (2010).

[34] Yan, Z.Y., Jiang, J.D.: Nonautonomous discrete rogue wave solutions and interactions in an inhomogeneous lattice with varying coefficients. J. Math. Anal. Appl 395, 542-549 (2012).

[35] Ablowitz, M.J., Segur, H.: Solitons and the Inverse Scattering Transform, SIAM, Philadelphia, (1981).

[36] Ablowitz, M.J., Ladik, J.: Nonlinear differential-difference equations. J. Math. Phys 16, 598-603 (1975). 
[37] Akhmediev, N., Ankiewicz, A.: Modulation instability, Fermi-Pasta-Ulam recurrence, rogue waves, nonlinear phase shift, and exact solutions of the Ablowitz-Ladik equation. Phys. Rev. E 83, 046603 (2011).

[38] Doktorov, E.V., Matsuk, N.P., Rothos, V.M.: Dynamics of the Ablowitz-Ladik soliton train. Phys. Rev. E 69, 056607 (2004).

[39] Hennig, D., Tsironis, G.P.: Wave Transmission in Nonlinear Lattices. Phys. Rep 307, 333 (1999).

[40] Yu, F.J.: Nonautonomous discrete bright soliton solutions and interaction management for the Ablowitz-Ladik equation. Phys. Rev. E 91, 032914 (2015).

[41] Cai, D., Bishop, A.R., Gronbech-Jensen, N.: Localized states in discrete nonlinear schrodinger equations. Phys. Rev. Lett 72, 591-595 (1994).

[42] Narita, K.: Soliton Solution for Discrete Hirota Equation. J. Phys. Soc. Jpn 59, 3528-3530 (1990).

[43] Mieck, B., Graham, R.: Bose-Einstein condensate of kicked rotators with time-dependent interaction. J. Phys. A 38, L139-144 (2005).

[44] Ablowitz, M.J., Ladik, J.F.: On the Solution of a Class of Nonlinear Partial Differential Equations. Stud. Appl. Math 57, 1-12 (1977).

[45] Vakhnenko, O.O., Vakhnenko, V.O.: Physically corrected Ablowitz-Ladik model and its application to the Peierls-Nabarro problem. Phys. Lett. A 196, 307-312 (1995).

[46] Yan, Z.Y.: Financial Rogue Waves Appearing in the Coupled Nonlinear Volatility and Option Pricing Model. Phys. Lett. A 375, 4274 (2011).

[47] Dai, C.Q., Zhang, J.F.: Exact spatial similaritons and rogons in 2D graded-index waveguides. Opti. Lett 35, 2651-2653 (2010).

[48] Yu, F.J.: Nonautonomous rogue waves and 'catch' dynamics for the combined Hirota-LPD equation with variable coefficients Commun. Nonlinear. Sci. Numer. Simulat 34, 142-153 (2016).

[49] Yu, F.J.: Multi-rogue waves for a higher-order nonlinear Schrodinger equation in optical fibers, APPL. MATH. COMPU 220, 176-184 (2013).

[50] Zhang, J.L., Wang, H.X.: Exact solutions and linear stability analysis for two-dimensional AlbowitzLadik equation. Chi. Phys. B 23(4), 044208 (2014).

[51] Wu, X.Y., Tian, B., Liu, L., Sun, Y.: Bright and dark solitons for a discrete (2+1)-dimensional Ablowitz-Ladik equation for the nonlinear optics and Bose-Einstein condensates. Commun. Nonlinear. Sci. Numer. Simulat 50, 201-210 (2017). 


\section{Figures}

(b)

(a)
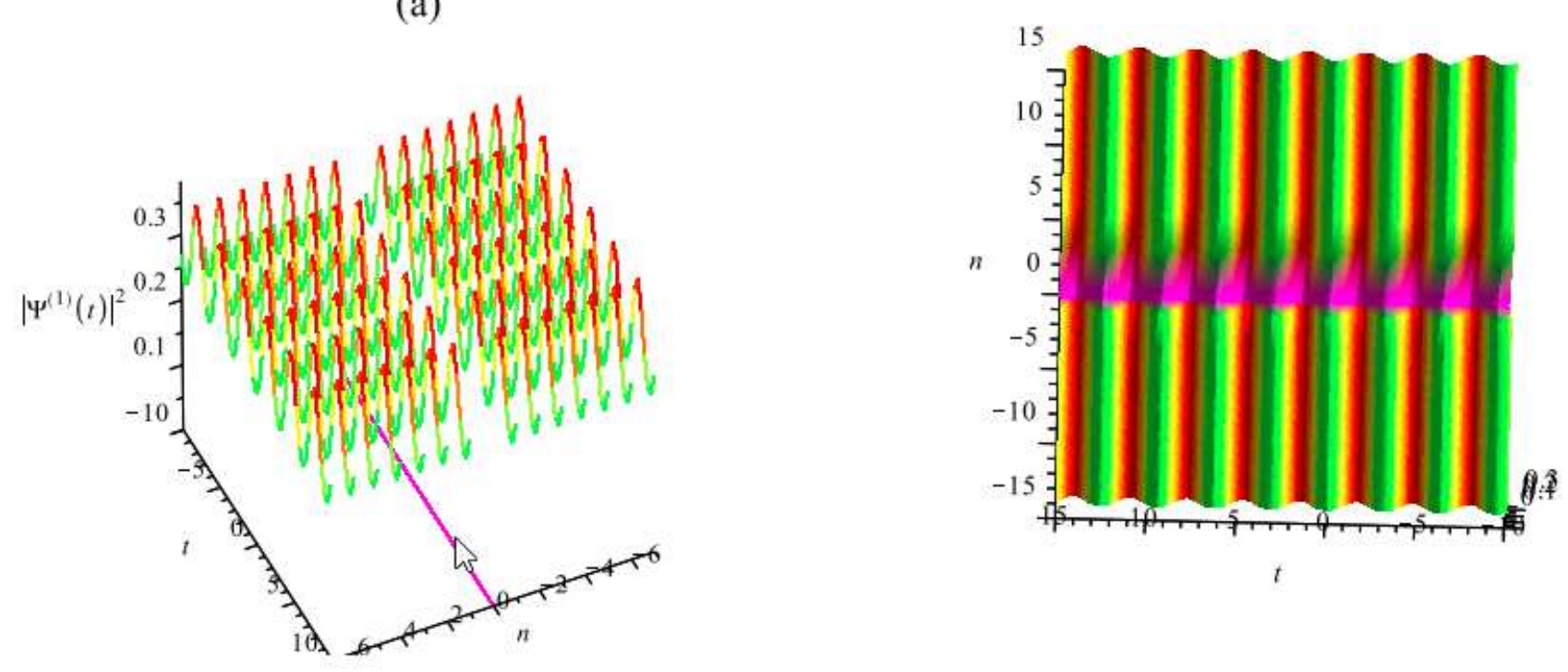

(d)

(c)
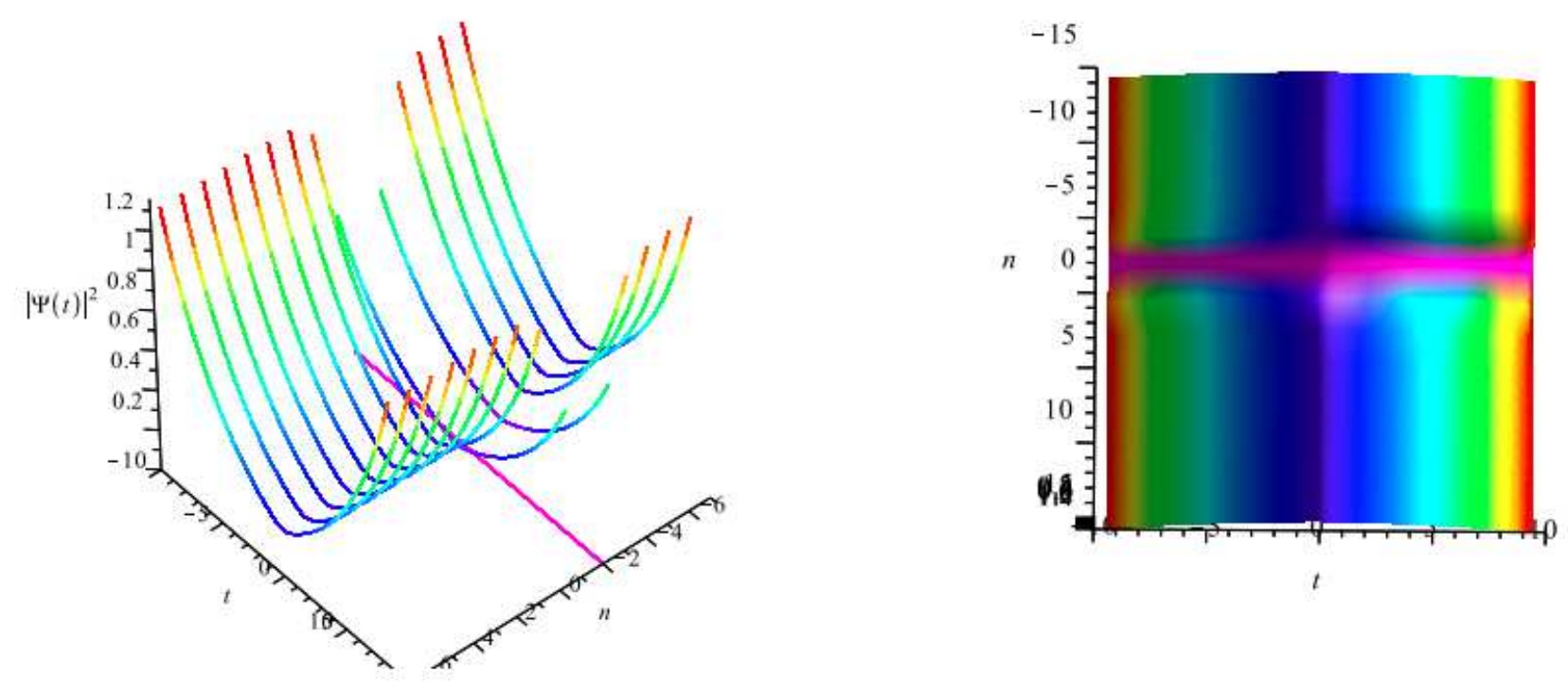

Figure 1

Please see the Manuscript PDF file for the complete figure caption 
(b)

(a)
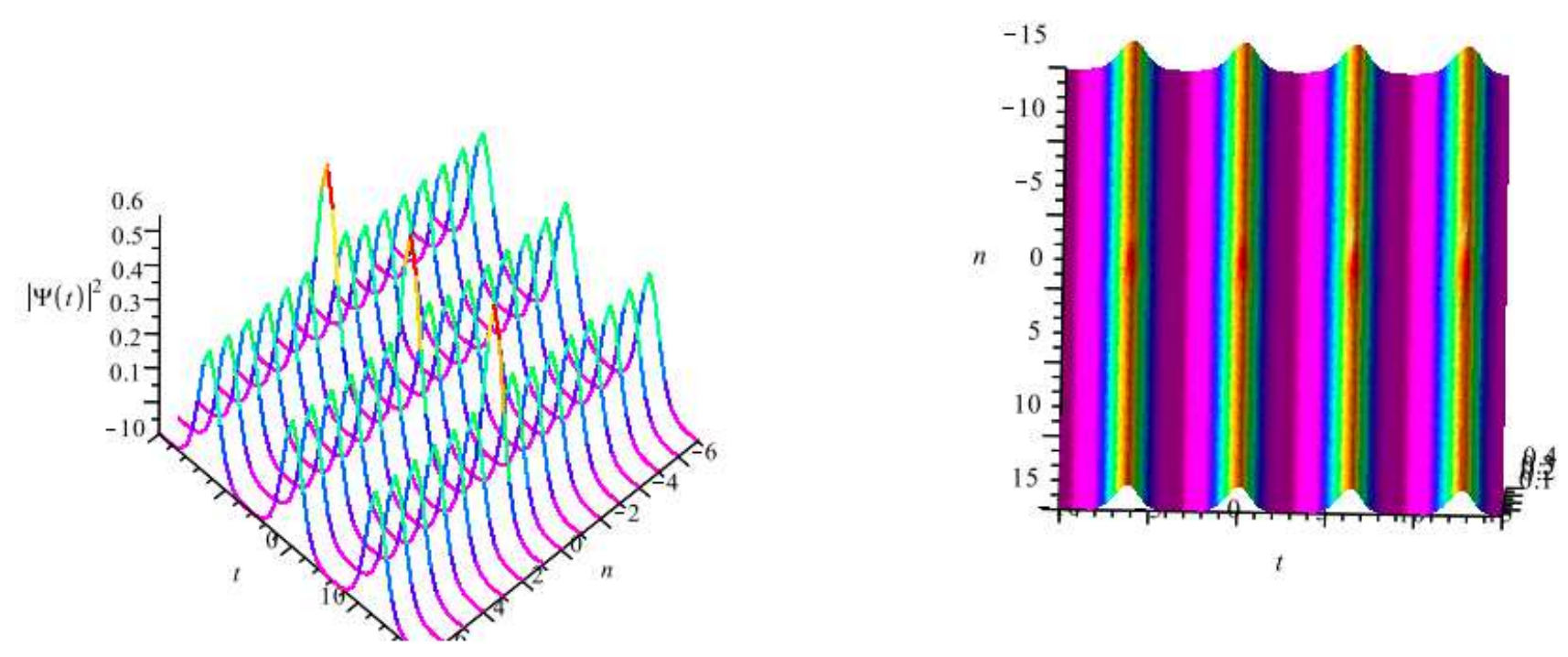

(d)

(c)
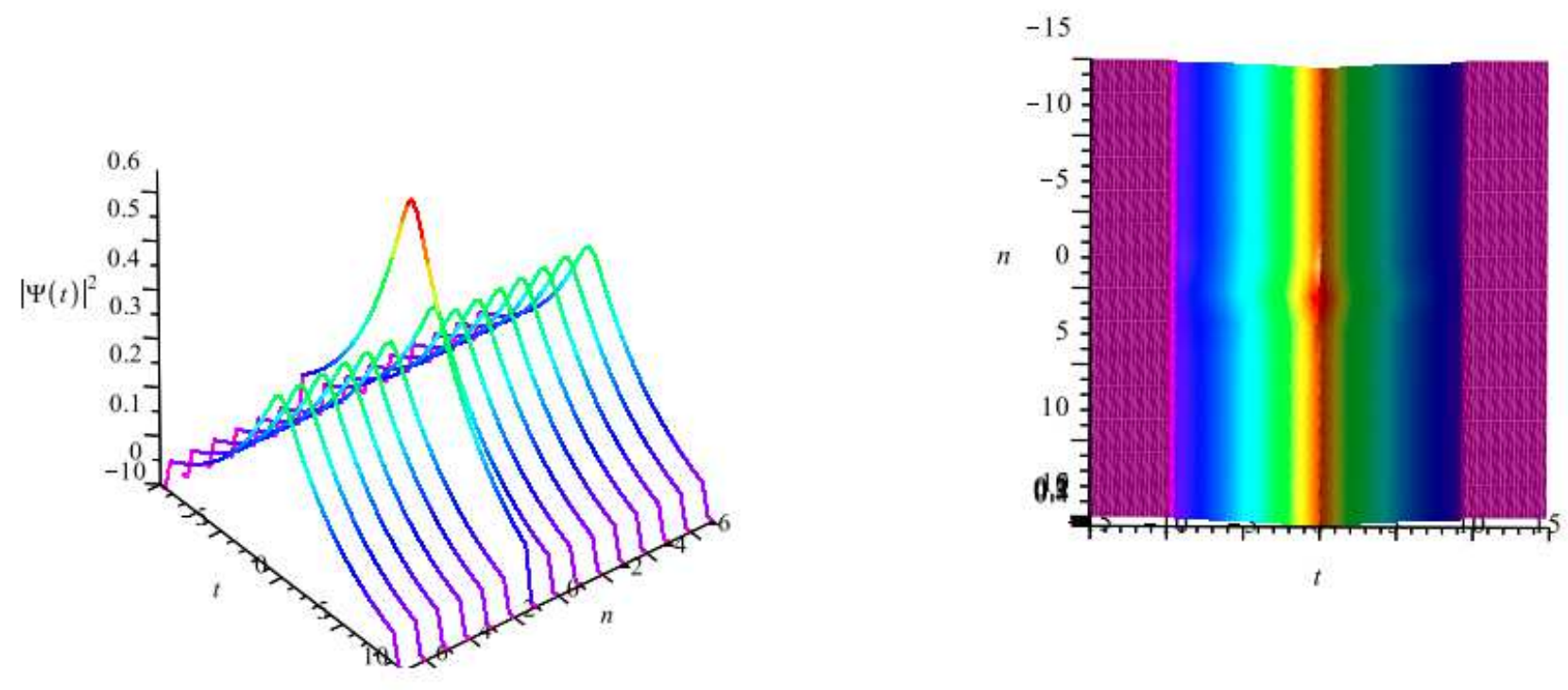

\section{Figure 2}

Please see the Manuscript PDF file for the complete figure caption 


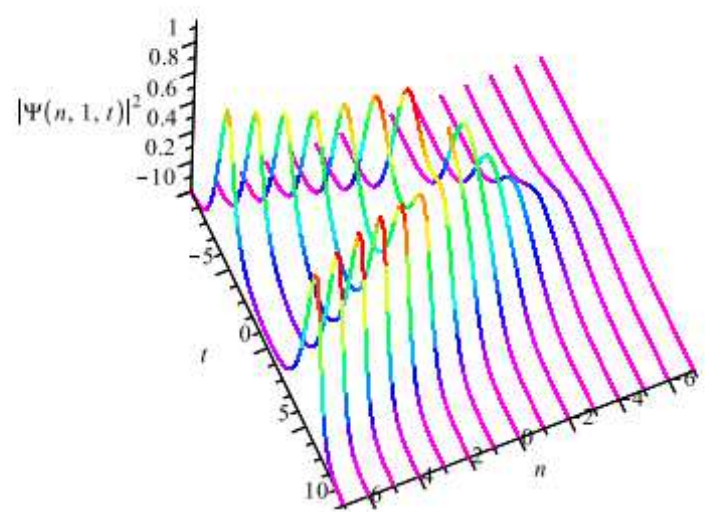

(c)

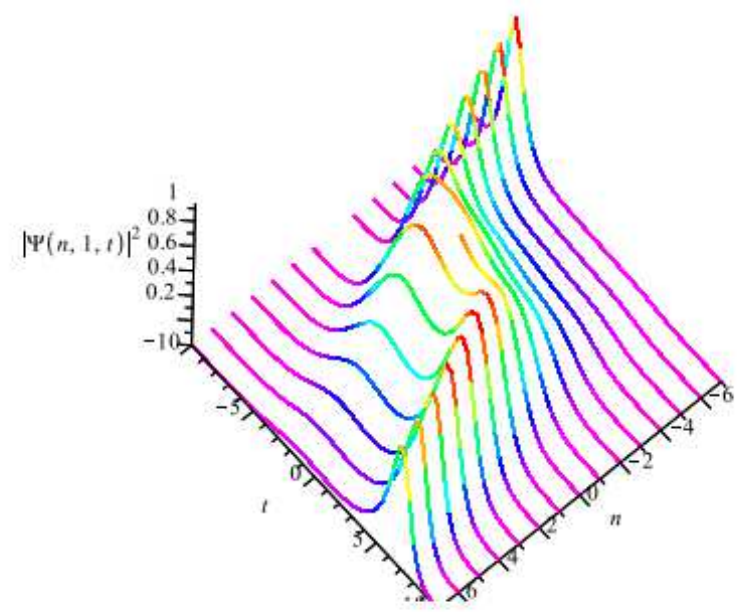

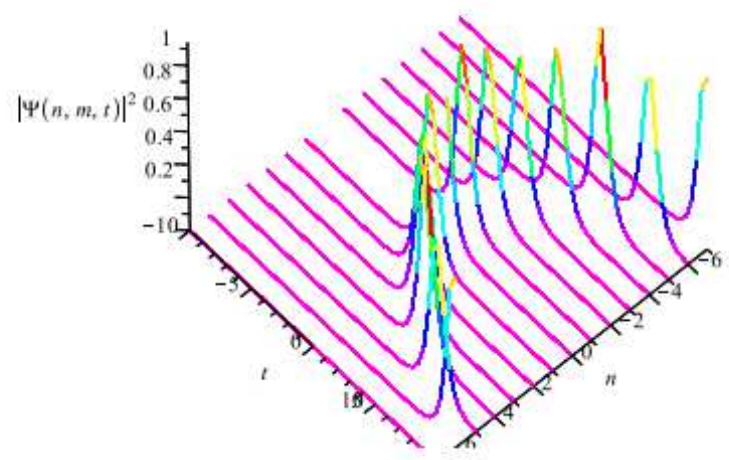

(d)

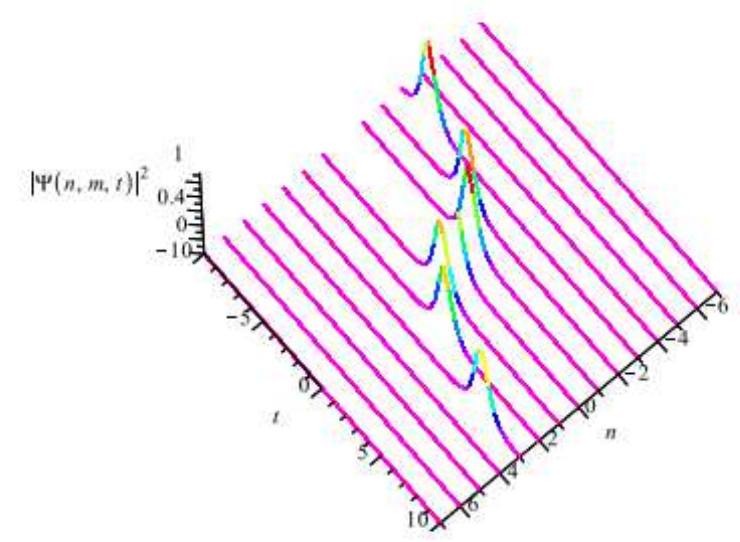

Figure 3

Please see the Manuscript PDF file for the complete figure caption 
(a)

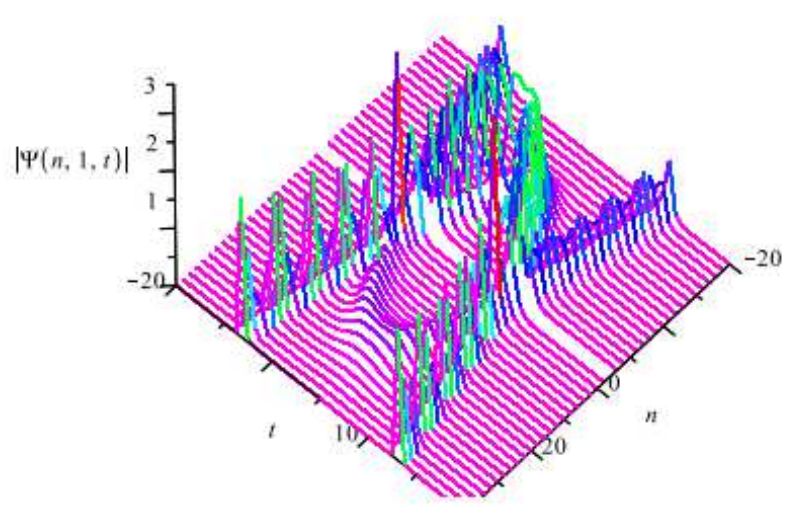

(c)

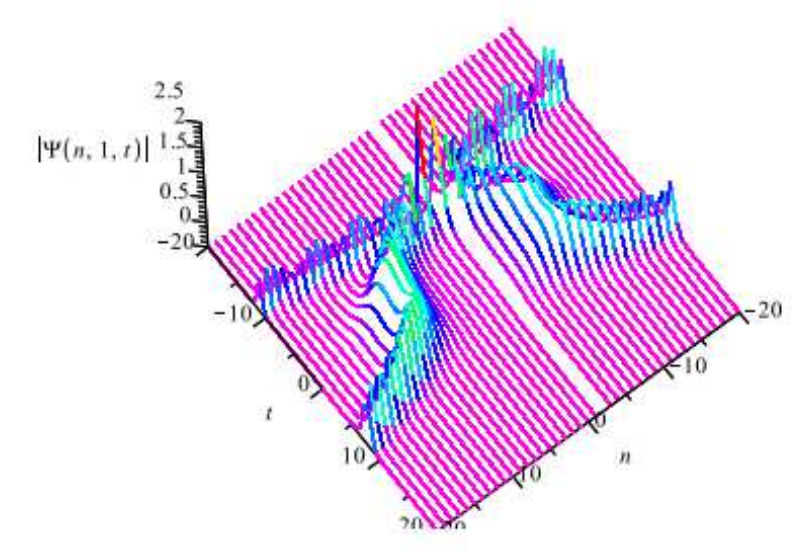

(b)

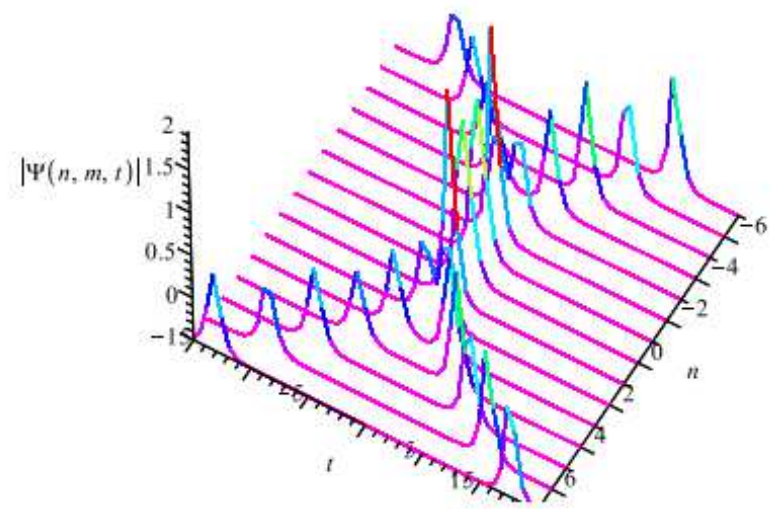

(d)

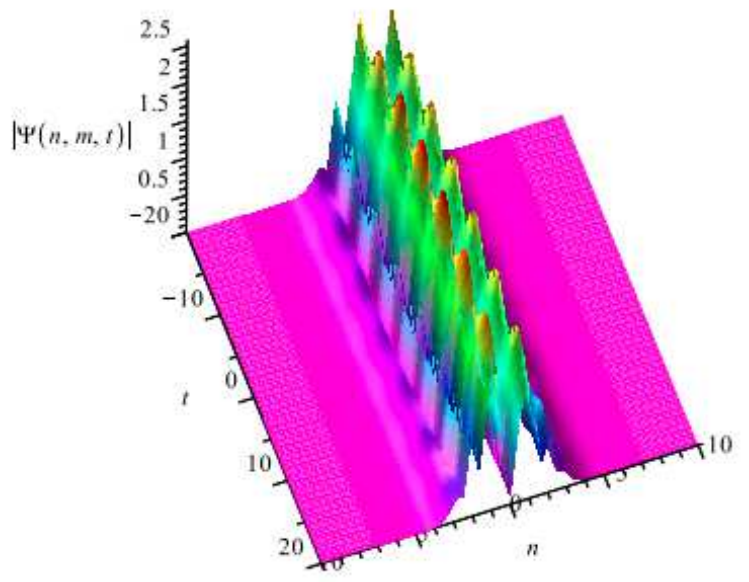

Figure 4

Please see the Manuscript PDF file for the complete figure caption 
(a)

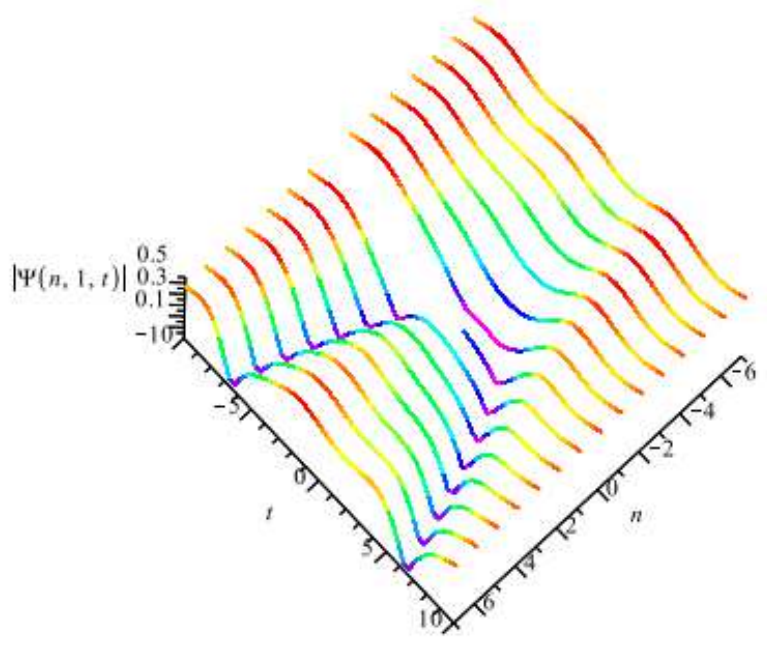

(c)

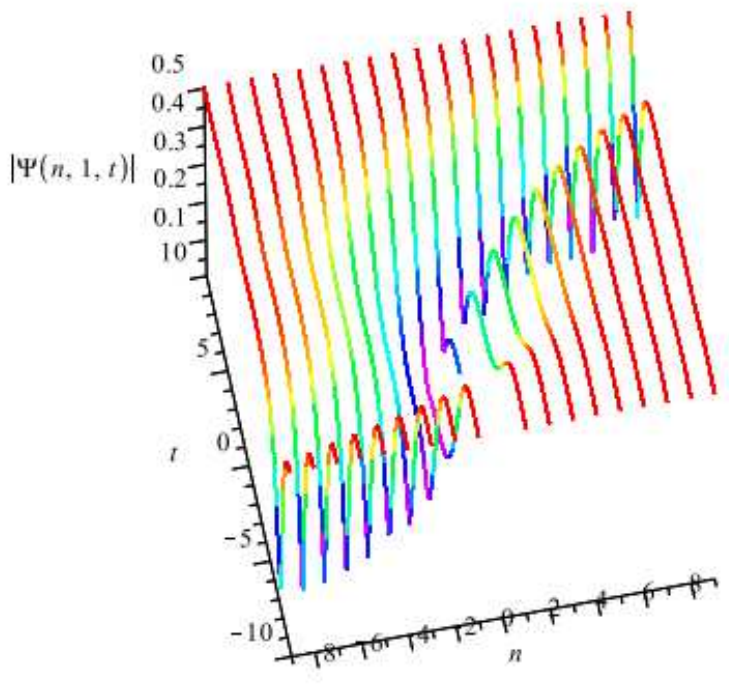

(b)

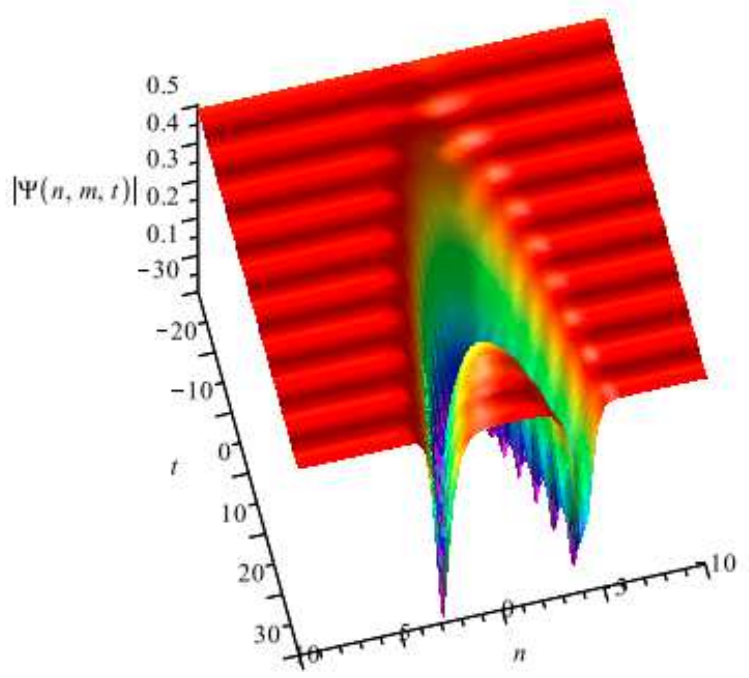

(d)

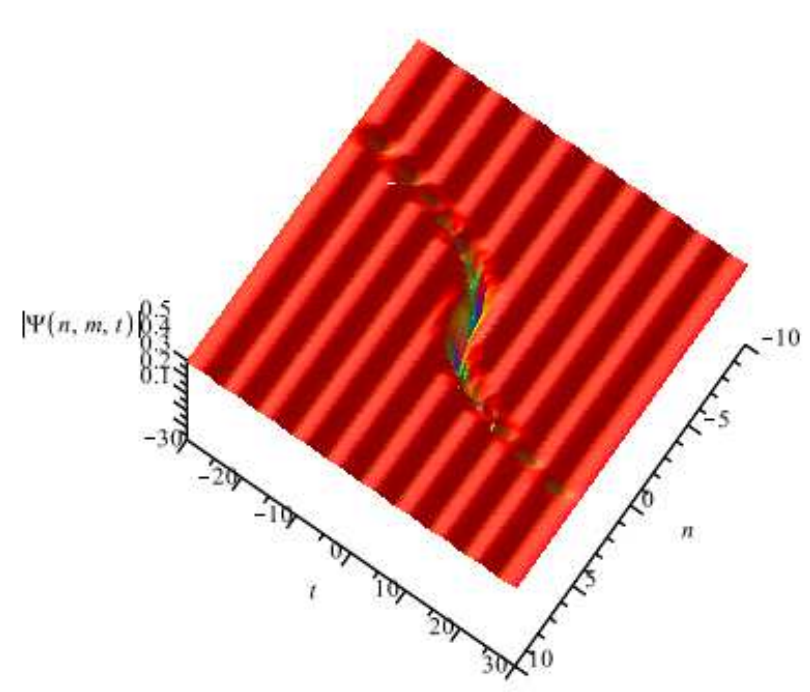

Figure 5

Please see the Manuscript PDF file for the complete figure caption 
(b)

(a)
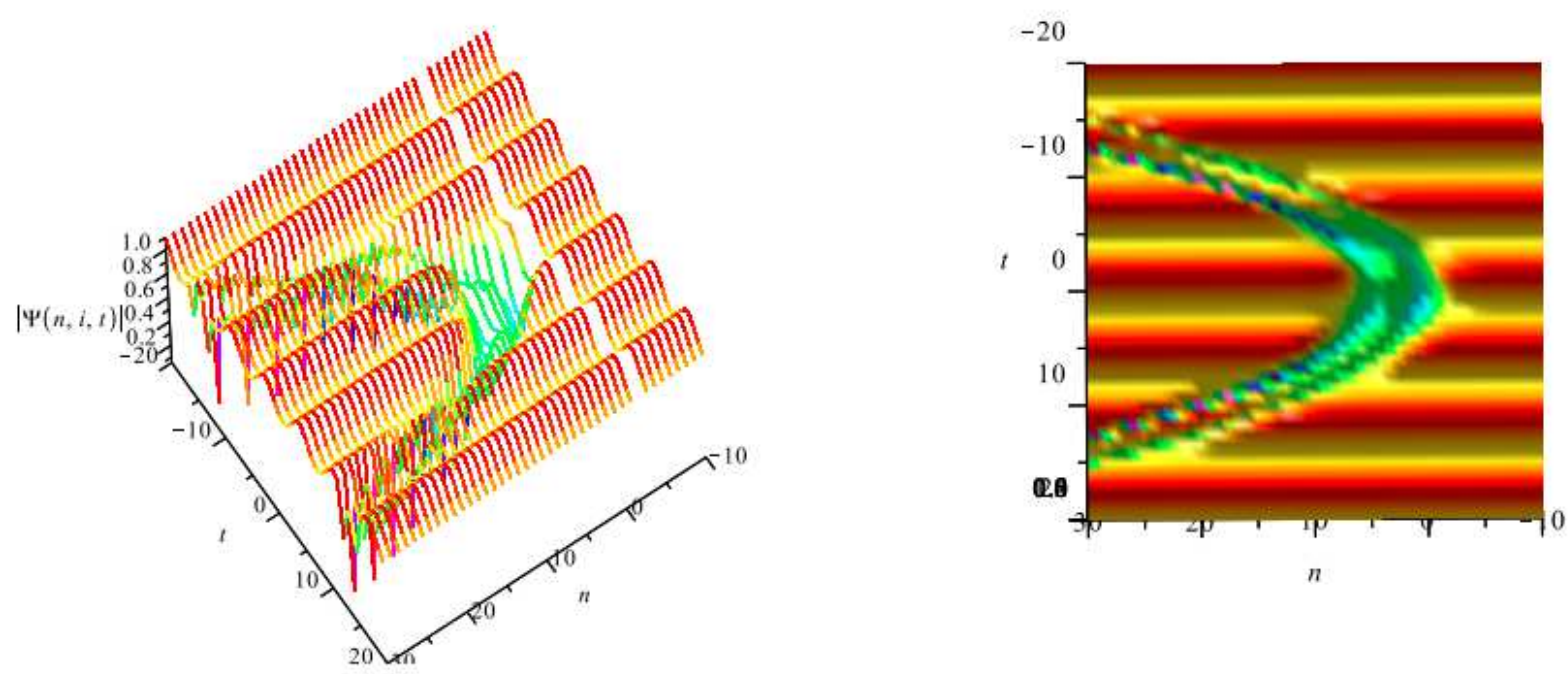

(d)

(c)
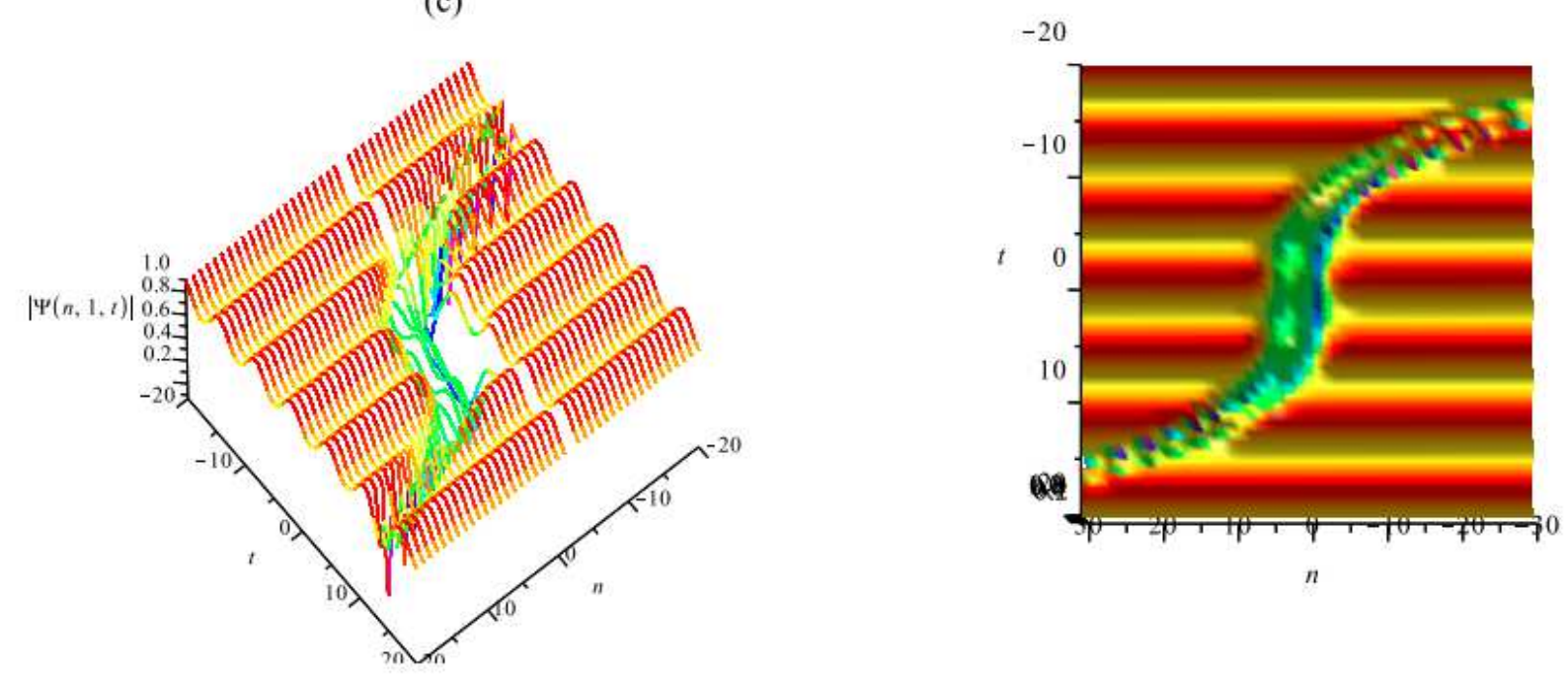

Figure 6

Please see the Manuscript PDF file for the complete figure caption 
(b)

(a)
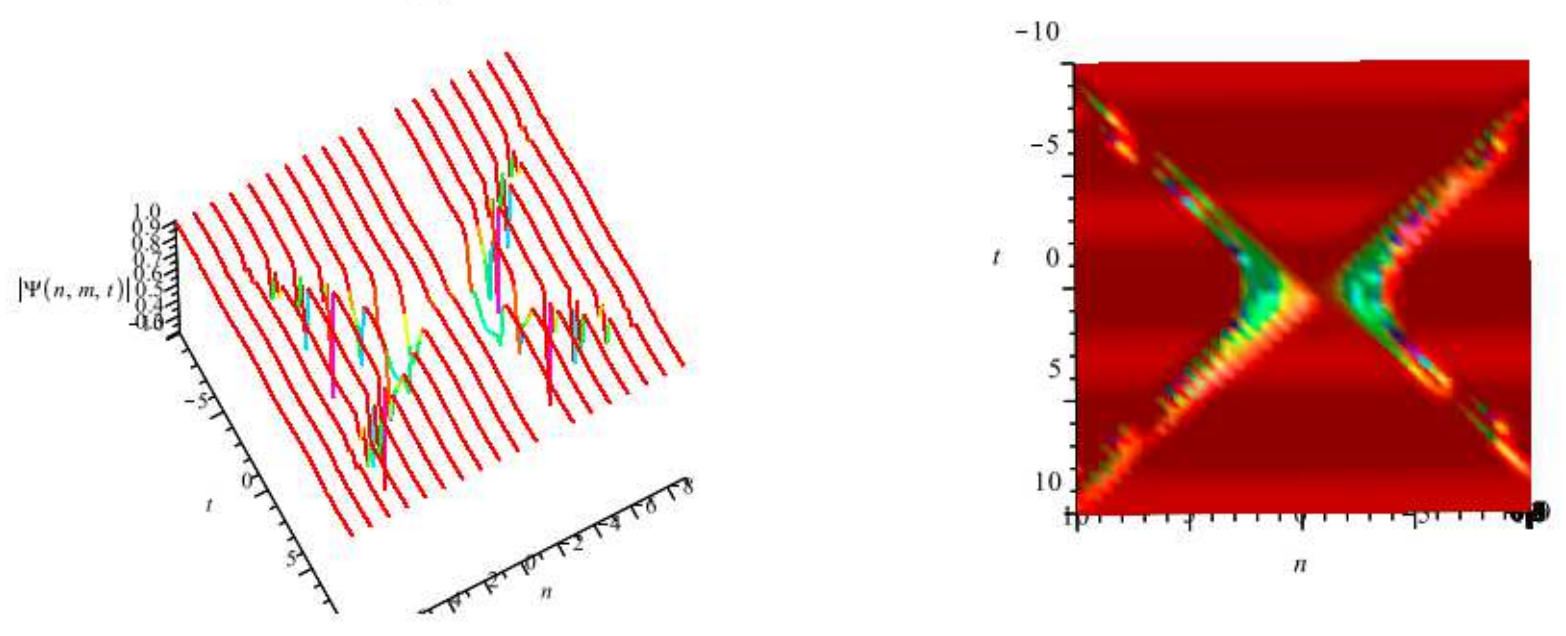

(d) (c)

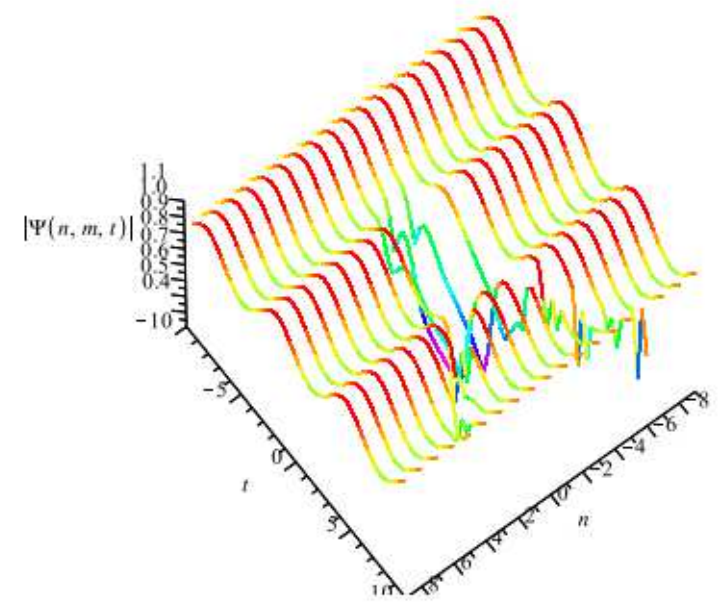

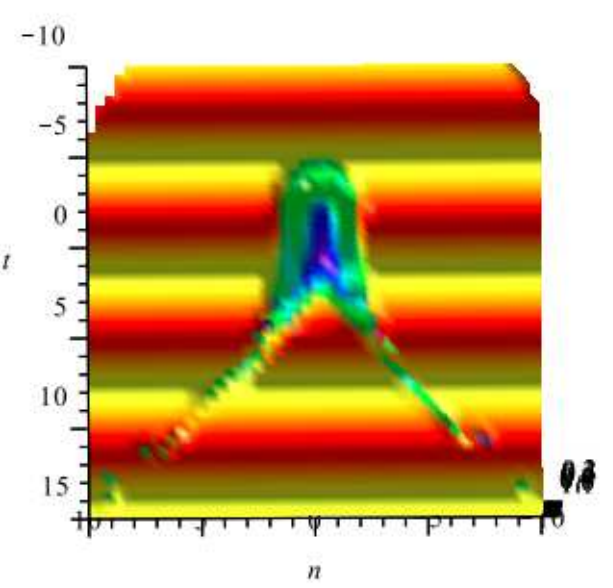

Figure 7

Please see the Manuscript PDF file for the complete figure caption 